\title{
Genus im DaF-Unterricht in Italien: Was sagen Lehrwerke und Grammatiken?
}

\author{
Marion Weerning (Palermo)
}

\begin{abstract}
For foreign language students gender seems to be a great problem. This article about the teachability and learnability of German gender wants to show what Italian students learn about it and how they do so (and also how they could do it better).
\end{abstract}

\section{$1 \quad$ Genusfehler und DaF-Unterricht}

Es wäre zu erwarten, dass das dreigliedrige Genussystem des Deutschen und die komplexe Überlagerung semantischer, morphologischer und phonologischer Genuszuweisungsregeln für DaF-Lerner ${ }^{1}$ ein enormes Problem darstellen. Doch nicht alle Beobachtungen bestätigen das. So hat sich z. B. bei einem großangelegten Projekt zum Deutschunterricht in der französischsprachigen Schweiz gezeigt, dass die Schüler viel weniger Genusfehler machen als erwartet (vgl. Christen 2000b: 175), was auch wir - Marie Rieger (vgl. ihren Beitrag in diesem Band) und ich - in unserer gemeinsamen Zeit als Dozentinnen am Goethe-Institut in der Spracharbeit und in der Lehrerfortbildung sowie in unserer heutigen Arbeit im universitären Bereich immer wieder festgestellt haben.

Dabei wäre natürlich zu diskutieren, was "wenige Fehler" heißt - immerhin liegt schon bei einer reinen Zufallsauswahl die Wahrscheinlichkeit, einem Substantiv das richtige Genus zuzuweisen, bei einem Drittel. Trotzdem gehen wir davon aus, dass ein hoher Anteil korrekter Genuszuweisungen in der Sprachproduktion nicht nur Glückssache ist, sondern dass die Lerner das Genus der Substantive richtig in ihrem mentalen Lexikon gespeichert haben und/oder dass sie Genuszuweisungsregeln anwenden. Begehen sie hingegen massiv Genusverstöße, so führen wir das darauf zurück, dass sie das Konzept Genus nicht verstanden haben.

Zur Überprüfung unserer Vermutungen haben wir eine empirische Untersuchung mit Studenten durchgeführt, die zeigen sollte, wie sicher sich die Lerner bei der Genuszuweisung fühlen, ob sie über ein gewisses Regelwissen verfügen (sowie ggf. über welches) und wie viele Genusfehler sie überhaupt machen (vgl. dazu Marie Rieger in diesem Band). Daneben haben wir an italienischen Schulen und Universitäten gängige Lehrwerke und didaktische Grammatiken ${ }^{2}$ untersucht, um herauszubekommen, wie Lernern dort Genus vermittelt wird (darum geht es in Kapitel 2 dieses Artikels), was sie dort über Genus und seine Funktionen sowie darüber, nach welchen Kriterien es zugewiesen wird, erfahren (s. Kapitel 3 und 4), und schließlich, was

\footnotetext{
${ }^{1}$ Wenn in diesem Artikel generisches Maskulinum benutzt wird, sind natürlich immer Männer und Frauen gemeint.

${ }^{2}$ Vgl. dazu im Anhang 2 die Liste der untersuchten Lehrwerke und Grammatiken. Es handelt sich dabei sowohl um von deutschen als auch von italienischen Verlagen herausgebrachte Werke sowie um Spezialausgaben von in Deutschland herausgegebenen Lehrwerken für den italienischen Markt. Dieser Artikel beschränkt sich auf den Unterricht DaF auf A1/A2-Niveau des Gemeinsamen Europäischen Referenzrahmens, weshalb nur jeweils die ersten Bände von jedem Lehrwerk untersucht wurden.
} 
Lehrwerke tun, um den Lernern dabei zu helfen, das richtige Genus mental zu speichern (s. Kapitel 5).

\section{$2 \quad$ Wie vermitteln Lehrwerke Genus?}

\subsection{Wie und wann führen Lehrwerke Genus ein?}

Lehrwerke sind im Idealfall effektiv und benutzerfreundlich und vermeiden jeden unnötigen theoretischen Ballast, denn der Aufwand, der für die Vermittlung grammatischer Beschreibungsmodelle erforderlich ist, muss in einem vertretbaren Verhältnis zum - ohnehin bisweilen fraglichen - Nutzen dieser Modelle stehen (vgl. Putzer 2004: 395, der sich auf Klotz 2004: 154 bezieht). Moderne Lehrwerke verfügen meist über einen praktischen Lehrbuchteil, der Wortschatz, Grammatik und kommunikative Funktionen einführt, sowie über einen Übungsapparat (oft separat als Arbeitsbuch) und über theoretische Erklärungen in Tabellenund/oder beschreibender Form.

In allen hier untersuchten Lehrwerken trifft der Lerner, wenn nicht schon in der allerersten Lektion, so doch in einer der ersten Lektionen, auf das Phänomen Genus. Dabei findet sich im Lehrbuch oftmals ein Text, in dem Artikel vor Substantiven stehen:

(1) Herzlich willkommen [...]! [Foto mit Untertitel] Bern die Altstadt [Foto mit Untertitel] Berlin das Brandenburger Tor [...] $\left(\mathrm{St}^{3:} 6\right)$

Andere Lehrwerke dagegen bringen Texte, in denen Personalpronomen vorkommen:

(2) [...] Familie Simpson. [...] Homer Simpson. Er ist [...]. [...] Marge Simpson. Sie ist [...]. [...] das Baby. Es heißt [...]. (VD-L: L17)

Nicht immer wird unmittelbar im Anschluss eine Thematisierung des grammatischen Phänomens Genus gegeben (wie in BP), sie erfolgt aber doch immer sehr bald.

Dass Genus schon im frühsten Stadium des DaF-Lernens eine Rolle spielt, ist nicht anders zu erwarten. Die Mehrzahl deutscher Sätze enthält schließlich "zumindest einen Artikel, ein derWort wie dies-, jed-, ein-, ein Wort wie mein-, dein-, ihr- usw., ein Pronomen oder ein Adjektiv, das sich auf ein entsprechendes Substantiv bezieht und dessen Kasus, Numerus und grammatisches Genus reflektiert" (Sperber 1989: 147). Substantive machen übrigens den größten Teil des deutschen Wortschatzes aus: laut Fleischer/Helbig/Lerchner (2001: 248) über die Hälfte, nach Engel (2004: 270) sogar 80\%.

Während in allen Lehrwerken in den allerersten Lektionen explizit gesagt wird, dass es im Deutschen Maskulinum (M), Femininum (F) und Neutrum (N) gibt, erscheint in den meisten in Deutschland veröffentlichten Lehrwerken weder in den Grammatikerklärungen noch im Sachregister der Terminus Genus (es werden z. T. nur Tabellen gebracht mit drei Spalten zu den drei Genera und Beispielen). Im Gegensatz zu den deutschen Lehrwerken tendieren dagegen die in Italien publizierten Lehrwerke und die Spezialausgaben in Deutschland herausgegebener Lehrwerke für den italienischen Markt dazu, den entsprechenden Begriff genere zu gebrauchen:

(3) In tedesco ci sono tre generi: maschile, femminile e neutro. [Übersetzung: ${ }^{4}$ Im Deutschen gibt es drei Genera: Maskulinum, Femininum und Neutrum.] (St: 9)

Die meisten Lehrwerke führen Maskulinum, Femininum und Neutrum zusammen mit dem Definitartikel ein (St, Di, VD-L/AB, BP, Eu, Op-L/AB). Dabei wird die Form des Artikels im

\footnotetext{
${ }^{3}$ Die im Folgenden benutzten Siglen werden im Anhang 2 erklärt.

${ }^{4}$ Alle Übersetzungen stammen von der Verfasserin.
} 
Nominativ oft als Genusmarker verwendet (so als seien der, die, das die Defaultformen eines Substantivbegleiters); BP schreibt etwa nicht Obst ist immer Femininum, sondern stattdessen:

(4) Obst hat immer den Artikel die (BP: 59) ${ }^{5}$

Andere Lehrwerke verfahren ähnlich, wenn sie sagen:

(5) Ormai sei abituato ad associare a ciascun sostantivo der, die oppure das. [Inzwischen bist du daran gewöhnt, mit jedem Substantiv der, die oder das zu assoziieren.] (VD-AB: AB25)

(6) Per i nomi l'appartenenza al genere è segnalato dall'articolo. [Für die Nomen wird die Genuszugehörigkeit durch den Artikel signalisiert.] (Ga: 25)

Einige Lehrwerke führen die Kategorien Maskulinum, Femininum, Neutrum zusammen mit Substantiven ein, die Nicht-Personen bezeichnen; d. h. sie führen direkt grammatisches Genus ein:

(7) der Turm, die Burg, das Rathaus (St: 8-9)

Andere dagegen beginnen mit Substantiven, die Personen bezeichnen, und lassen somit die Kategorie Genus als nach biologischem Geschlecht semantisch motiviert erscheinen. Di z. B. bringt, nachdem in Lektion 1 schon er als maskulines Personalpronomen der dritten Person und sie als feminines Personalpronomen der dritten Person eingeführt wurde (ibd.: 8), in Lektion 2 nur Personenbezeichnungen aus dem Wortfeld Familie und fügt hinzu:

(8) Finora hai incontrato solo due generi: maschile (der Vater) e femminile (die Mutter). In tedesco esiste un altro genere, il neutro, che incontrerai più avanti. [Bis jetzt hast du nur zwei Genera angetroffen: Maskulinum (der Vater) und Femininum (die Mutter). Im Deutschen gibt es ein weiteres Genus, das Neutrum, auf das du später treffen wirst.] (ibd.: 15)

Di beginnt also mit Sexus (d. h. natürlichem oder biologischem Geschlecht); Ga u. a. beziehen dabei auch ein sexusindifferentes Neutrum ein:

(9) der Graphiker, die Graphikerin, das Fotomodell (Ga: 25)

Alle Lehrwerke bieten Tabellen, die den Formenbestand der Artikel und Pronomen, also den morphologischen Aspekt der Genusmarkierung, darstellen.

Ferner weisen alle Lehrwerke in ihren Grammatikerklärungen oder mit einem Lerntipp darauf hin, dass jedes Substantiv zusammen mit seinem Artikel memorisiert werden sollte: ${ }^{6}$

(10) Lerntipp: Nomen immer mit Artikel lernen (Eu: 14)

Die meisten empfehlen im Zusammenhang mit der Pluralbildung, jedes Substantiv zusammen mit seinem Artikel und der Pluralform zu lernen:

(11) Quando si studia un vocabolo tedesco occorre quindi memorizzare, oltre al genere, anche la forma del plurale. [Wenn man eine deutsche Vokabel lernt, muss man, neben dem Genus, auch die Pluralform memorisieren.] (Di: 37)

\subsection{Wie differenzieren Lehrwerke Genus und Sexus?}

Nur wenige Lehrwerke thematisieren den Unterschied zwischen Genus und Sexus, so wie es z. B. Ga tut:

(10) il genere grammaticale non sempre coincide con il genere naturale [grammatisches Geschlecht stimmt nicht immer mit natürlichem Geschlecht überein] (Ga: 25)

Die Lehrwerke, die Genus über Sexus einführen, lassen vermuten, dass Genus etwas mit Sexus zu tun hat, ${ }^{7}$ sagen das aber nie ausdrücklich. Auch die Lehrwerke, die direkt grammati-

\footnotetext{
${ }^{5}$ Es müsste eigentlich heißen: "Die Bezeichnungen für Obst(sorten) haben immer den Artikel die".

${ }^{6}$ Diesen Lerntipp hat es schon in der Renaissance gegeben (so Kelly 1969: 46, zitiert nach Sperber 1989: 225).
} 
sches Genus einführen, beziehen keine klare Position: Sie sagen nicht explizit, dass Genus im Endeffekt bloß ein Sortierungsverfahren ermöglicht, nach dem das Deutsche seine Substantive in drei Gruppen ordnet (so wie andere Sprachen ihre Substantive in nur zwei oder auch in mehr als zehn Gruppen einteilen). ${ }^{8}$

Auch in dem Moment, wo allgemeine Personenbezeichnungen eingeführt werden, die im Deutschen fast immer maskulin sind, auch wenn Frauen mitgemeint sind (wie in In meiner Klasse sind 20 Schüler), wird in den Lehrwerken nicht auf die Problematik Genus/Sexus hingewiesen. Es wird weder hervorgehoben, dass beim generischen Maskulinum das semantische Merkmal [+männlich] neutralisiert ist (vgl. Ulrich 1988: 391f.) und Genus und Sexus nicht kongruent sind, noch wird gesagt, dass hier nur Männer genannt und Frauen ausgeschlossen werden.

Dabei sorgt diese sprachliche Problematik seit Jahrzehnten für Diskussionsstoff in der deutschsprachigen Öffentlichkeit. Schließlich haben die Studien der sog. feministischen Linguistik dazu beitragen, dass es inzwischen normal ist, das generische Maskulinum durch alternative Formulierungen $\mathrm{zu}$ ersetzen, die Frauen nicht mehr diskriminierend ausschließen (z. B. indem man allgemein von Lernenden oder genauer von LernerInnen statt nur von Lernern redet), denn generisches Maskulinum macht Frauen unsichtbar - so schon Trömel-Plötz (1978), Kalverkämper (1979), Pusch (1979) u.a. Empirische Studien z. B. von Braun/Scezny/Stahlberg (2002: 79-84) zeigen, dass der gedankliche Einschluss von Frauen beim generischen Maskulinum (Nennen Sie einen Politiker/drei Politiker) tatsächlich geringer ist, als wenn die Frauen explizit genannt werden (Nennen Sie drei PolitikerInnen). ${ }^{9}$

All diese Aspekte werden in den Lehrwerken weder unter landeskundlichem Aspekt noch unter sprachlichen Gesichtspunkten beleuchtet.

\section{$3 \quad$ Wozu dient Genus?}

Was Genus ist und wozu es dient, wird in keinem Lehrwerk explizit gesagt. Es wird anscheinend vorausgesetzt, dass die Lerner die Kategorie aus dem schulischen L1-Unterricht kennen. So beginnt die Erste Lektion eines seit über 60 Jahren immer wieder neu aufgelegten italienischen Lehrwerks von Alella/Marini (1991: 17) so:

\footnotetext{
${ }^{7}$ Wie weit Sexus etwas mit Genus zu tun hat, beschäftigt die Linguisten schon lange. Wegera (1997: 14) meint, dass "sich die patriarchalisch bestimmte Geschichte unserer Sprache auch in der Genusvergabe niedergeschlagen hat".

Das Thema sorgte schon in der Vergangenheit für Diskussionsstoff. Während z. B. Grimm (1890) in der Genuszuweisung eine Übertragung männlicher und weiblicher Merkmale sieht, wird diese These schon von z. B. Brugmann (1889) angefochten, für den Genus auch sprachgeschichtlich nicht primär Sexus-Ausdruck ist (mehr zu diesen beiden Positionen u. a. bei Sieburg 1997: 9).

${ }^{8}$ Aber wenn Genus nicht Sexus ausdrückt, was drückt es dann aus? Für Leiss z. B. stellt es eine Quantifikationskategorie dar, was auch im Einklang steht mit Greenbergs Universal 36: "If language has a a category of gender, it always has a category of number" (Denning/Kemmer 1990: 58). "Die Numeruskategorie stellt die ersten paradigmatisch wählbaren Quantifikationstypen der nominalen Referenz bereit. Der Plural spezifiziert den Referenten als aus identischen Teilen bestehendes unbegrenztes Ganzes. Die Genuskategorien erweitern und differenzieren das Spektrum der Quantifikationstypen" (Leiss 2005: 13f.). In diese Richtung geht auch Werner (2008): Maskulinum = Singulativum [+ zählbar] und [-kollektiv], Femininum = Kollektivum/Abstraktum [+ zählbar] und [+kollektiv], Neutrum $=$ Kontinuativum [+masse] und [-kollektiv].

${ }^{9}$ In diesem Artikel wird das traditionelle generische Maskulinum beibehalten und von Sprachlernern geredet, weil die Verfasserin der Meinung ist, dass in den Köpfen der potentiellen Leser dieses Artikels Sprachlerner mit Sicherheit beiden Geschlechtes sind. Wäre die Sprache von Politikern, würde sie es zweifelsohne vorziehen, von PolikerInnen zu sprechen, um sicher zu gehen, dass der potentielle Leser auch wirklich Frauen in seinem Kopf miteinbezieht.
} 
(13) 1. Nella lingua tedesca vi sono:

tre generi: maschile, femminile, neutro

due numeri:

[1. In der deutschen Sprache gibt es

drei Genera: Maskulinum, Femininum, Neutrum

zwei Numeri:]

$[\ldots]$

Dass es hier um nominale Kategorien geht, wird erst unter Punkt 2 klar:

(14) 2. Non vi è una regola fissa per determinare il genere dei sostantivi

[2. Es gibt keine feste Regel, um das Genus der Substantive zu bestimmen] [...] (ibd.)

Ob Genus eine semantische Kategorie oder eine nomeninhärente Eigenschaft und somit eine grammatisch-lexikalische Kategorie ${ }^{10}$ ist, wird in den Lehrwerken nicht geklärt. Außerdem wird nirgendwo die syntaktische Funktion von Genus zur "Sicherung des grammatischen Zusammenhangs der Satzglieder (Kohäsion)" (Heupel 1973: 81) explizit herausgearbeitet, obwohl der Lerner laufend mit Texten konfrontiert wird, aus denen sie ableitbar ist. So legt das Genus des Nomens auf der einen Seite intraparadigmatisch das Genus von NP-internen Bezugseinheiten - letzter Schultag (Ba: 146) - und auf der anderen Seite interparadigmatisch das Genus von NP-externen Bezugseinheiten - [...] ein Drucker. Der Drucker ist ja billig [...]. Der ist bestimmt kaputt. (BP: 27) - fest. Es dient also einerseits der Konstituentenbildung und andererseits der Textreferenz (vgl. Hoberg 2004: 5f.). Während auf die Konstituentenbildung eingegangen wird, wird auf die Textreferenz - auch in den modernen Lehrwerken (ibd.: 6) - kaum hingewiesen: weder durch graphische Hervorhebung im Text mit Pfeilen z. B. vom Pronomen auf das Substantiv, auf das es sich bezieht (wie allerdings in Op-L: 12), noch durch explizite Nennung (wie jedoch in Op-L: 12, 61, 76).

Zwar hat Wegener (1995: 10) recht, wenn sie behauptet, "Genusverstöße führen praktisch nie zu Missverständnissen" (so ist der Satz Ich habe *einen Katze und *eine Hund ohne Probleme verständlich). Sobald wir aber die paradigmatische Ebene verlassen und Pronomen ins Spiel kommen - wir uns also auf die syntagmatische Ebene begeben -, dann kann falsches Genus sehr wohl zu Missverständnissen führen (Ich habe *einen Katze und *eine Hund. Stell dir vor, es [?] ist größer als er [**der Katze?] ist unklar, während Ich habe eine Katze und einen Hund. Stell dir vor, sie ist größer als er unmissverständlich ist).

Genus ist also wichtig; den Lernern muss bewusst gemacht werden, wozu es dient: Genus ist eine grammatische Kategorie, "die nicht wie andere eine einzige globale Funktion hat, es sei denn man fasse seine vielfältigen gegenwärtigen Aufgaben im Standarddeutschen als eine Art 'Jokerfunktion' zusammen" (Fischer 2005: 339f.), die Zusammengehöriges kennzeichnet. Es wäre wünschenswert, wenn in den Lehrwerken klarer herausgestellt würde, welche Funktion Genus nicht nur innerhalb der Nominalphrase, sondern auch im Text hat. Schließlich können Genusmarkierungen genauso wichtig sein wie die "Rückennummern von Spielern auf einem Fußballfeld" (Bates et al. 1995: 848, zitiert nach Menzel 2004: 35).

Positiv hingegen fällt auf, dass einige Lehrwerke kontrastiv Bezug aufs Italienische als L1 nehmen:

(15) In tedesco ci sono tre generi: il maschile e il femminile, come in italiano, e il neutro [Im Deutschen gibt es drei Genera: Maskulinum und Femininum, wie im Italienischen, und Neutrum] [...] (VD-AB: AB5)

\footnotetext{
${ }^{10}$ So Bußmann (2008: 227); für Leiss (2005: 13) dagegen ist Genus nur Teil des Lexikons und nicht der Grammatik, während Welte (1974: 145) es definiert als "sprachspezifische, grammatische 'Kategorie' des Nomens, die sich 'morphosyntaktisch' [...] manifestieren kann".
} 
Darauf hinzuweisen, dass Genuszugehörigkeit in den beiden Sprachen nicht unbedingt übereinstimmt, ist zweifelsohne wichtig, denn "der prägende Einfluss der L1 für den L2-Erwerb ist inzwischen unbestritten" (Christen 2000a: 28). Dies tut z. B. Di:

(16) Attenzione! Non c'è sempre corrispondenza tra il genere di un sostantivo in italiano e in tedesco [Achtung! Es herrscht nicht immer Übereinstimmung zwischen dem Genus eines Substantivs im Italienischen und im Deutschen] (la sedia $\rightarrow$ der Stuhl). (ibd.: 36)

Einige Lehrwerke nehmen auch kontrastiv Bezug aufs Englische, da Englisch für 97\% der italienischen Schüler ${ }^{11}$ die erste Fremdsprache darstellt, die somit schon vor Deutsch gelernt wird und als Quelle für Interferenzfehler nicht zu unterschätzen ist.

(17) A differenza dell'inglese, i sostantivi indicanti oggetti inanimati non sono necessariamente neutri (es.: die Schule) e il neutro può essere usato anche per esseri animati (es.: das Kind). [Im Unterschied zum Englischen sind die Substantive, die unbelebte Gegenstände beschreiben, nicht unbedingt Neutra (Bsp.: die Schule) und das Neutrum kann auch für belebte Wesen gebraucht werden (Bsp.: das Kind)]. (Op-AB: 17)

Mehr theoretisches Wissen zum Thema Genus bringen die wenigsten Lehrwerke und auch nicht unbedingt die didaktischen Grammatiken.

\section{$4 \quad$ Welche Genuszuweisungsregeln gibt es?}

Auf Eisenbergs (2006b: 150) Frage "Nach welchen Kriterien wird einem Substantiv des Deutschen ein grammatisches Geschlecht zugewiesen und welcher Gebrauch wird vom Genus gemacht?" antwortet kein Lehrwerk. Einige Lehrwerke behaupten sogar:

(18) Per imparare il genere dei sostantivi non esistono regole precise [Um das Genus von Substantiven zu lernen, gibt es keine genauen Regeln] (Op-AB: 17).

Dass es keine oder nur wenige Regeln zur Genusbestimmung gibt, ist ein Standpunkt, den fast alle sog. didaktischen Grammatiken vertreten sowie auch einige wissenschaftliche Grammatiken. Weinrich (2005: 325) meint: "Für das heutige Sprachbewußtsein ist das Genus der meisten Nomina [...] willkürlich. Man muß es als grammatische Tatsache mit dem Nomen lernen". Für ihn ist Genus eine "für den Sprecher der Sprache 'blind' vorgegebene Kategorie" (ibd.: 326). Und Helbig/Buscha (2001: 245f.) beginnen ihr Kapitel zum grammatischen Genus so: "Im Rahmen einer Darstellung der Gegenwartssprache ist es für die Mehrzahl der Substantive nicht möglich, praktikable Regeln zur Genusbestimmung zu geben".

Genus wird also oft als arbiträr dargestellt. Deshalb haben die Lerner (und auch die Lehrer) ganz einfach das Gefühl, es sei - und zwar "ausgangssprachenunspezifisch" (Götze 1984: 4) "ein erhebliches Lehr- und Lernproblem" (ibd.: 2) oder gar als für Deutschlerner "eine der größten Lernschwierigkeiten in der Grammatik" (Krohn/Krohn 2008: 10) und eine der "bedeutendsten Fehlerquellen im DaF-Unterricht" (Wegera 1996: 9).

Dabei könnte man Genus doch auch von vornherein anders präsentieren, so wie es auch die Mehrheit der wissenschaftlichen Standardgrammatiken macht, und die Kategorie Genus lernbarer erscheinen lassen: "Ein Großteil der deutschen Substantive wird durch Ableitung aus anderen Wörtern mit Hilfe von Ableitungssuffixen gewonnen. [Diese] bestimmen fast immer ihr grammatisches Geschlecht." (Eisenberg 2006b: 151). Oder: "Genuszuordnung erfolgt im Deutschen primär nach formalen Kriterien wie Wortausgang [...], Einsilbigkeit [...], nur vereinzelt nach der Semantik" (Wegener in Barkowski/Krumm 2010: 100).

"Il genere di un determinato sostantivo è, al contrario di quanto si ritiene comunemente, tutt'altro che casuale [Das Genus eines bestimmten Substantivs ist, im Gegensatz zu dem, was

\footnotetext{
${ }^{11}$ Angabe des italienischen Bildungsministeriums:

http://www.pubblica.istruzione.it/dg_studieprogrammazione/index:new .shtml, Stand Januar 2007.
} 
allgemein angenommen wird, alles andere als zufällig]" (Di Meola 2004: 62), denn es gibt valide Regeln. ${ }^{12}$ Diese Regeln werden oftmals auch von den gleichen Autoren, die am Anfang des Lehr-/Lernprozesses pessimistisch das Genus als willkürlich erscheinen ließen, zu einem späteren Zeitpunkt mehr oder weniger ausführlich genannt: Das gilt für die Lehrwerke, die in den Folgebänden genusbestimmende Regeln auflisten, für die eben zitierten Weinrich und Helbig/Buscha und für den Großteil der didaktischen Grammatiken, von denen allerdings ein Drittel (DS, SM, SS, FD) weder Zuweisungsregeln noch andere Erklärungen zur Funktion des Genus liefert.

\subsection{Genuszuweisungsregeln mit 100\%-iger Gültigkeit}

Entgegen den Erwartungen tauchen nicht alle Genuszuweisungsregeln, die 100\%-ige Gültigkeit haben, in den Lehrwerken und didaktischen Grammatiken auf.

Auf die Derivationsregeln "Nomina agentis auf -er $\rightarrow$ M" (M1a, b) ${ }^{13}$ und "Nomina agentis auf -in $\rightarrow \mathrm{F}^{\prime \prime}$ (M2) weisen fast alle Lehrwerke hin. Das hat sicherlich damit zu tun, dass in einem kommunikativen Anfängerunterricht die Sprachhandlung "Kontaktaufnahme" inkl. "Vorstellung" schon am Anfang einen wichtigen Platz einnimmt, zu deren Realisierung viele Nomina agentis wie Berufsbezeichnungen gebraucht werden.

Die Derivationsregel "Diminuitiva auf -chen und -lein $\rightarrow$ N" (M3) dagegen wird nur in wenigen Lehrwerken erwähnt (dann meist nur, wenn das Wort Mädchen eingeführt wird, um zu erklären, warum es Neutrum ist). Das mag daran liegen, dass es nicht sehr viele Wörter mit diesen Suffixen auf A-Niveau gibt.

Die Derivationsregel "Substantive auf -ung, -heit etc. $\rightarrow$ F" (M4) findet sich meist erst in den Folgebänden der Lehrwerke - vielleicht weil es zumindest auf A1-Niveau nicht sehr viele Substantive gibt, die diese Endungen haben. Andere Derivationsregeln (M1c, M1d, M5, M6) erscheinen trotz ihrer 100\%-igen Validität nicht in Lehrwerken auf A-Niveau.

Was die didaktischen Grammatiken betrifft, die Genusregeln bringen, so werden die eben genannten Derivationsregeln (M1-4) normalerweise mehr oder weniger komplett genannt. Es überrascht, dass die Derivationsregel "Substantive aus Ge- + Verbstamm + $-e$ " (M5) und die Konversionsregel "substantivierte Infinitive $\rightarrow$ N" (M6) trotz ihrer enormen Produktivität überhaupt nicht (M5) bzw. kaum (M6 nur bei He und FD) erscheinen.

\subsection{Genuszuweisungsregeln mit fast $100 \%$-iger Gültigkeit}

Kommen wir zu den Regeln mit fast 100\%-iger Gültigkeit. Die Sexus-Regel "Bezeichnungen für männliche Personen $\rightarrow \mathrm{M}$, Bezeichnungen für weibliche Personen $\rightarrow \mathrm{F}^{\prime \prime}$ (S1a) und die Default-Genus-Regel für Personenbezeichnungen "Personenbezeichnungen $\rightarrow$ M" (S1b) sog. generisches Maskulinum - werden weder in den Lehrwerken noch in den meisten didaktischen Grammatiken auseinander gehalten. Dass es ein generisches Maskulinum gibt, darauf weisen nur $\mathrm{Ga}$ bei den Lehrwerken und He bei den didaktischen Grammatiken hin. Es wird teilweise sogar nicht einmal auf die Sexus-Regel aufmerksam gemacht (LP, WM, FT).

Auch weitere Genusregeln zur Morphologie (M8-10 und M11-15) finden sich - bis auf die Schwa-Regel "Substantive auf $-e \rightarrow$ meist F" (M7) - trotz ihrer hohen Validität kaum in den Lehrwerken.

Die Untersuchung der didaktischen Grammatiken, die Genuszuweisung behandeln, ergibt dagegen: Die Schwa-Regel bringen fast alle (bis auf FD), "Substantive auf -er $\rightarrow$ meist M" (M9) ebenfalls (bis auf Lu), "Substantive auf -en $\rightarrow$ meist M" (M8) die meisten (bis auf LP,

\footnotetext{
${ }^{12}$ Detailliert dazu u. a.: Duden (2006: 154-68), Fischer (2005: 87-134), Hoberg (2004: 85-109).

${ }^{13}$ In Klammern steht der Verweis auf die kommentierte Regel in Anhang 1 dieses Artikels.
} 
Rei, He, FD), während "Substantive auf -el $\rightarrow$ meist M" (M10) fehlt (außer bei WM) wie auch "Substantive mit Präfix Ge- $\rightarrow$ meist N" (M11, fehlt außer bei BF). Genannt werden hingegen die auf A-Niveau weniger relevanten Regeln zu den Substantiven auf -ling, -nis, tum, den Deverbativa auf - $t$ und ohne Suffix (M12-15: M12 zweimal, M13 -nis sowie -tum je viermal, M14 dreimal, M15 zweimal).

Nirgendwo werden den Lernern Lernhilfen angeboten, die das Memorisieren der in den Regeln angetroffenen Suffixe erleichtern. Dabei könnten sich Abwandlungen von Sperbers mnemotechnischem Vorschlag (1989: 152), sich statt mehr als einem Dutzend verschiedener Suffixe nur drei Quasi-Wörter - "der Iglingorismus, die Heitungkeiteischaftion, das Tumchenmamentum"14 - einzuprägen, bestimmt als nützlich erweisen.

\subsection{Weitere Genuszuweisungsregeln}

\subsubsection{Zuweisungsregeln nach Deklinationsklassen}

Wenn Lehrwerke und Grammatiken über Deklinationsklassen im Deutschen sprechen, liegt es in der Natur der Sache, auch auf Genus zu sprechen zu kommen. Da aber der Genitiv des Substantivs in der Unterrichtspraxis normalerweise erst auf B-Niveau als letzter der vier Kasus eingeführt wird, kann die Genitiv-Regel "Genitivendung nein $\rightarrow$ F" (M16) auf A-Niveau kaum Anwendung finden (dabei ist sie sowieso recht unbefriedigend, da sie eine riesige Restklasse von Maskulina und Neutra nicht miteinschließt).

Dagegen wird der Plural schon in den ersten Lektionen eingeführt, was erlaubt, Genus und Plural in Verbindung zu bringen, denn "Pluralmarkierung ist wesentlich vom Genus abhängig" (Thieroff/Vogel 2009: 40). Doch nur wenige Lehrwerke (Ba, VD-L/AB) weisen explizit darauf hin, während die meisten diese Tatsache völlig unter den Tisch fallen lassen. In den Lehrwerken und Grammatiken wird immer - wenn überhaupt - von der Genusklasse (also Substantive im Singular) auf die Pluralklasse geschlossen, aber nie umgekehrt, so wie es hingegen im Anhang (M17) dargestellt wird.

Ferner laden schon im DaF-Unterricht auf A-Niveau die Tabellen zur Deklination von Artikelwörtern und Substantiven dazu ein, die Ähnlichkeit zwischen Maskulinum und Neutrum herauszuarbeiten ${ }^{15}$ (M: dem Mann, des Mannes; N: dem Kind, des Kindes), was aber nirgendwo explizit gemacht wird.

Für italienischsprachige DaF-Lerner ist eine Korrelation zwischen Genus und Deklinationsklassen nichts Neues, da es sie auch im Italienischen gibt (sehr detailliert dazu: Lima et al. i. Vorb.). Auch wenn sich im Italienischen klarer als im Deutschen viele Substantive von ihrer Deklinationsklasse ausgehend in eine Genusklasse einordnen lassen, ${ }^{16}$ so sind auch im Italienischen immerhin fast ein Drittel der Substantive ${ }^{17}$ nicht über die Deklinationsklasse klassifizierbar. ${ }^{18}$ Die Erwähnung dieser Tatsache könnte sich im Unterricht als vorteilhaft erweisen, um das leider so verbreitete Vorurteil "Deutsche Sprache - schwere Sprache (die anderen Sprachen sind viel einfacher)" zu entschärfen.

\footnotetext{
14 -ig, -ling, -or, -ismus; -heit, -ung, -keit, -ei, -schaft, -ion; -tum, -chen, -ma, -ment, -um.

${ }^{15}$ Thieroff/Vogel (2009: 50) fassen die beiden Genera zum "'Super-Genus' der Nicht-Feminina" zusammen.

${ }^{16}$ Substantive auf - $a$ sind fast immer feminin, auf - $o$ fast immer maskulin.

${ }^{17}$ Zählungen zu den sechs Flexionsklassen im Italienischen gibt es bei Chini (1998).

${ }^{18}$ Dies gilt für die zur -e/-i-Klasse gehörenden wie maskulines il fiore $\rightarrow i$ fiori, feminines la siepe $\rightarrow$ le siepi und die mit unverändertem Plural wie maskulines $i l r e \rightarrow i$ re, feminines la città $\rightarrow$ le città.
} 


\subsubsection{Semantische Zuweisungsregeln}

Neben den beiden schon behandelten Regeln zu Sexus und generischem Maskulinum (S1a, b) gibt es weitere semantische Regeln. In didaktischen Grammatiken werden diese Regeln oft gemischt mit den morphologischen aufgelistet, in die Lehrwerke finden sie dagegen kaum Eingang. Auf A-Niveau könnten allerdings die fast ausnahmslos geltenden Regeln "Tageszeiten, Wochentage etc. $\rightarrow$ M" (S2a), "Niederschläge $\rightarrow$ M" (S2b), "Alkoholika $\rightarrow$ M" (S2c) nützlich sein. Andere semantische Regeln betreffen einen auf A-Niveau nicht relevanten Wortschatz oder haben zu viele Ausnahmen.

Auch hierzu hat Sperber (1989: 152) eine Lernstrategie bereit: Abgrenzend zu den QuasiWörtern für morphologische Endungen (s.o.) schlägt er für das Einprägen semantischer Aspekte Merkverse vor, die "durch Reim und Rhythmus mehr Lerngewinn bringen als das ständige Wiederholen einer Liste". Sperbers Idee wird aber von keinem Lehrwerk aufgenommen. Ein denkbarer Merkspruch wäre:

Giorni, parti del giorno, mesi e stagioni

sono per i masculoni.

Ma la notte

è delle ragazzotte.

[Freie Übersetzung: Tage, Tageszeiten, Monate und Jahre sind für die Machos gemacht.

Aber den Mädels gehört wenigstens die Nacht.] ${ }^{19}$

Während die ebengenannten semantischen Regeln Eingang in einige didaktische Grammatiken finden (S2a in LP, Rei, Lu, S2b in LP, Rei, S2c in He), gilt das nicht für andere semantische Genuszuweisungsprinzipien (S3-5), was an den zahlreichen Ausnahmen liegen mag. Wenn man sich die vielen Ausnahmen und die große Zahl der semantischen Klassen, ${ }^{20}$ wie sie aus den divergenten Auflistungen in den wissenschaftlichen Grammatiken hervorgehen, anschaut, so wundert es nicht, wenn Zifonun (2002: 8) resigniert feststellt: "Die Disparatheit solcher Klassen [Anmerkung: solch semantischer Klassen] zeigt [...], dass von einer genusprägenden Wirkung semantischer Klassenbildung nicht die Rede sein kann".

Auch phonologische Regeln finden keinen Eingang in Lehrwerke und didaktische Grammatiken, während neuere wissenschaftliche Grammatiken die diesbezüglichen Erkenntnisse von Köpcke und Zubin einbauen. Allerdings gehen hier die Meinungen stark auseinander: vom optimistischen Eisenberg (2006b, 152), für den für ungefähr 9 von 10 einsilbigen Substantiven das Genus unter Berücksichtigung phonologischer Gegebenheiten richtig voraussagbar ist, bis zu Hoberg (2004: 98), die meint: "Die Regeln zum Zusammenhang von phonologischer Struktur und Genus müssen [...] als relativ schwach gelten". Sicher ist, dass es sich nicht mehr um Regeln, sondern nur noch um Tendenzen handelt, wenn man formuliert: "Einsilber $\rightarrow$ oft M" (Ph3) o. ä.

\section{4 Kognitive Mechanismen bei der Genuszuweisung}

Während Muttersprachler, die über ein stabiles mentales Lexikon verfügen, über Reproduktion das gespeicherte Genus abrufen und nur selten in Verlegenheit kommen, ein Genus bewusst selegieren zu müssen, befinden sich L2-Lerner laufend in dieser Situation. Hierbei können sie aber unmöglich alle bisher genannten Regeln und Tendenzen in Bruchsekundenschnelle abrufbar haben. Aber irgendein Genus muss zusammen mit dem Substantiv selegiert werden: Was passiert also im Kopf des Lerners?

\footnotetext{
${ }^{19}$ Der Merkspruch stammt von der Verfasserin.

${ }^{20}$ Die Regeln sind in jeder wissenschaftlichen Grammatik nachzulesen, wobei es allerdings keine zahlenmäßige und inhaltliche Übereinstimmung zwischen den verschiedenen Handbüchern gibt. Aufgrund ihrer didaktischen Irrelevanz werden hier weitere semantische Ansätze wie die von Köpcke/Zubin (1983 etc.) auch nicht aufgegriffen.
} 
Gibt es da vielleicht auch ein "Default-Genus, das zugewiesen wird, wenn kein anderer Mechanismus greift" (Di Meola 2007b: 87f.)? Für Steinmetz (1986: 214) gibt es eine Genushierarchie: $\mathrm{M}>\mathrm{F}>\mathrm{N}$. Wenn keine Regeln zur Anwendung kommen können, dann ist ein Substantiv also maskulin. Anderer Meinung ist da Di Meola (2007b), der davon ausgeht, dass es das Neutrum ist, das zumindest für marginal im (mentalen) Lexikon integrierte Bildungen wie Fremdwörter (Handy) und andere Wörter, die selten zusammen mit Genusmarkern gebraucht werden (Tennis), als Default-Genus fungiert. Ob es ein spezifisches Default-Genus für italienische DaF-Lerner gibt, bliebe zu untersuchen.

In Zweifelsfällen kommt sicherlich auch das "Entlehnungsprinzip" (Köpcke/Zubin 1984: 89) zum Zuge, wonach das Genus eines phonetisch (die Stunde, Blume, *die Funke) und/oder semantisch ähnlichen (das Wasser, das Bier, *das Milch ${ }^{21}$ ) Substantivs oder des entsprechenden Substantivs aus der Muttersprache (italienisch la lettera feminin, *die Brief) gewählt wird.

\section{$5 \quad$ Wie trainieren Lehrwerke Genus?}

Unterscheiden wir mit Fischer (2005: 135-152 und 339) ${ }^{22}$ folgende Phasen der Genusetablierung:

- Genuszuordnung: Sie erfolgt beim ersten Kontakt durch Imitation (der Lerner hört oder liest z. B. das Haus).

- Genusspeicherung: Nach der Genuszuordnung kann das Genus im mentalen Lexikon des Lerners zusammen mit dem Substantiv über den Genusmarker das gespeichert werden (der Lerner speichert z. B. das Haus; natürlich können auch Morpheme gespeichert werden, Z. B. das Ge-).

- Genusabruf: Nach der Genusspeicherung kann das Genus zusammen mit dem Substantiv aus dem mentalen Lexikon (wo es sich im optimalen Fall richtig stabilisiert hat) abgerufen werden (der Lerner "weiß" jetzt, dass es das Haus heißt und braucht nur zu reproduzieren).

Wenn wir damit einverstanden sind, dass es diese drei Phasen gibt, dann müssen wir eigentlich zu der Erkenntnis kommen, dass die Genuszuordnung durch Imitation einen sehr großen Stellenwert im Unterricht haben muss, damit das Genus über den Definitartikel als Genusmarker richtig mitgespeichert wird und dann bei Bedarf richtig abgerufen werden kann. Damit es auch auf Dauer gespeichert bleibt, ist es didaktisch sinnvoll, dass das zu lernende Substantiv zusammen mit seinen genusmarkierenden Elementen (Artikeln, pränominalen Adjektiven) möglichst oft und abwechslungsreich trainiert wird, denn je mehr Arten der Erklärung angeboten werden, je mehr Kanäle der Wahrnehmung benutzt werden, desto besser wird Wissen gespeichert, desto vielfältiger wird es verankert und auch begriffen und erinnert (Vester 1975: 54). Diese Erkenntnis ist nicht neu und sicherlich allen Lehrbuchautoren bekannt. Daher überrascht es, wie wenig Übungen und Übungstypen es trotz alledem zum Genuserwerb in den Lehrwerken gibt.

Wenn Wörter nur über Texte gelernt werden, legt das nicht gerade lernerfreundliche Flexionssystem des Deutschen - sechs Artikelendungen -Ø, -(e)r, -e, -(e)s, -(e)n,-em²3 für 16 ver-

\footnotetext{
${ }^{21}$ Ob die Studenten in Marie Riegers Studie (Marie Rieger in diesem Band) wirklich aus diesem Grund *das Milch sagen, kann nur eine Hypothese sein.

${ }^{22}$ Für Fischer (2005: 341) ist Genuserwerb intraindividuelle Genusetablierung. Die gegenwärtige Genusverteilung deutscher Nomina ist dagegen nichts anderes als "das summarische Ergebnis der vielen einzelnen Genusetablierungsprozesse" (ibd.).

${ }^{23}$ Man kann auch von acht Endungen ausgehen, wenn man -ie und - $e$ sowie -as und -es separat sehen will (so wie z. B. Christen 2000b: 168).
} 
schiedene Genus-Numerus-Kasus-Kombinationen ${ }^{24}$ - dem Lerner schon die ersten Steine in den Weg:

Für Lernende ergibt sich aus der deutschen Formenökonomie rsp. der daraus resultierenden Polysemie einerseits ein erhebliches Analyseproblem beim Input (nur wenn das Genus von Schule bekannt ist, kann der Schule als Dativ interpretiert werden!), andererseits ein Syntheseproblem beim Output (an welcher syntaktischen Stelle muss welche Endung realisiert werden?). (Christen 2000b: 169)

Treffen die Lerner in einem Text z. B. auf aus der Schule, kann es passieren, dass sie fälschlicherweise den Schluss ziehen, Schule sei maskulin (da es Usus ist, den Definitartikel als Genusmarker anzusehen). So besteht die Gefahr, dass sie diesen Chunk unreflektiert lernen und dann den Artikel übergeneralisierend anwenden (*Der Schule ist neu). Deshalb erscheint es angebracht, dass Lerner im Anschluss an diese Begegnung auch an einer anderen Stelle (z. B. bei den Übungen im Arbeitsbuchteil) möglichst mehrfach mit der Form die Schule konfrontiert werden und sie dann selbst imitierend benutzen.

Wie sehen nun die (wenigen) Genusübungen aus, die in die untersuchten Lehrwerke aufgenommen wurden? Erst einmal fällt auf, dass nicht jedes Lehrwerk in dem Moment, wo es über Genus spricht, auch Übungen dazu anbietet. VD-L/AB oder WB führen Genus, wie im Inhaltsverzeichnis angekündigt, in Lektion 1 ein und liefern dort auch theoretische Erklärungen (VB-L/AB spricht dort sogar über das Suffix -in), aber die erste Arbeitsbuchübung kommt erst Lektionen später.

Eine typische Übung zum Genus, aus der auch intuitiv abzulesen ist, dass Genus eine "Kategorie zur nominalen Klassenbildung" (Bußmann 2008: 227) ist, ist das Ordnen einer vorgegebenen Gruppe von Substantiven in eine Tabelle mit drei Spalten - jeweils mit Maskulinum, Femininum, Neutrum betitelt. Eine unkonventionellere Variante dieses Übungstyps ist die Ankreuzübung:

1 der Apfelstrudel $[\mathrm{M}][\mathrm{F}][\mathrm{N}][\mathrm{Pl}]$

$2[\ldots] \quad[\mathrm{M}][\mathrm{F}][\mathrm{N}][\mathrm{Pl}]$

(Ga: 25)

In diese Richtung gehen auch Lerntipps, die den Lerner zu einer systematischen Einbeziehung des Genus beim Wortschatzlernen anhalten:

(20) Strategien. Ordina i sostantivi di ogni lezione secondo il loro genere: scrivi quelli maschili con una penna blu, quelli femminili con una penna rossa e quelli neutri con la matita. [Ordne die Substantive jeder Lektion nach ihrem Genus: Notiere die Maskulina mit einem Blaustift, die Feminina mit einem Rotstift und die Neutra mit einem Bleistift.] (Ba: AB28)

Das Hervorheben des Genus durch verschiedene Farben im Lehrbuch praktizieren auch St und Di. Sperber (1989: 169) merkt zum Einsatz der Farben an, dass es aber nicht ausreicht, Genera durch Farben zu konkretisieren, sondern dass eine Interaktionskomponente hinzugefügt werden müsse (z. B. interaktive Gedächtnisbilder wie der blaue Elephant, die rote Tür, das grüne Brot).

BP setzt bei der Wortschatzarbeit auf Artikelbilder (Zuordnen von Substantiven zu drei Bildern, z. B. der Tisch, das Bild, die Vase; BP: 207) und auf Wortschatzkarten (auf jede Karte schreibt der Lerner auf die Vorderseite das Wort mit Artikel und Pluralendung und ein Beispiel - der Kuli, -s: Das ist ein Kuli. - und auf die Rückseite die Übersetzung in die Muttersprache; BP: 39). Letzteren Tipp geben auch VD-AB und Op-L; Op-L rät zusätzlich, Karteikarten in drei verschiedenen Farben je nach Genus zu benutzen (Op-L: 17).

\footnotetext{
${ }^{24} 4$ Kasus x 4 (nämlich 3 Genera im Singular + 1 Plural mit Genusneutralisation).
} 
Genus kann auch spielerisch trainiert werden wie in Ba: AB25:

(21) Trova i sostantivi che si nascondono nel "serpentone" e scrivili nella colonna giusta. [Finde die Substantive, die in der Wortschlange versteckt sind, und schreibe sie in die richtige Spalte.]

AUGEAUTOBROTBUCHBUSFENSTERHUNDKATZEKUGELSCHREIBERLEHRER[...]

\begin{tabular}{|c|c|}
\hline der & 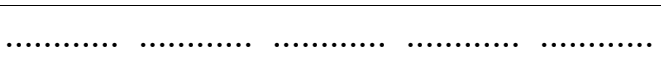 \\
\hline die & 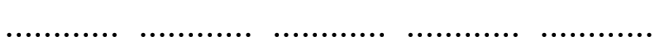 \\
\hline das & 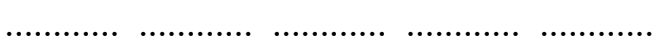 \\
\hline
\end{tabular}

Eine weitere Idee, um den Artikel als Genusmarker anzuwenden, findet sich in Ba: 40, wo ein Lerner aufgefordert wird, seine Hand in eine Stofftasche zu stecken, in der sich verschiedene bekannte Gegenstände befinden, und diese durch Tasten zu erraten und dann zu benennen (der Kuli). Kreativer gibt sich SD: 32, wo sich Lerner Artikelgeschichten ("ein Film im Kopf") ausdenken sollen, und lustiger als eine Lehrbuchübung ist sicher auch das Artikel-Quiz in Op-AB: 17: Jeder Lerner hat Wortkarten, A nennt ein Substantiv ohne Artikel (Ausgang?) und B antwortet mit dem passenden Artikel (der!). Ansonsten halten sich die Lehrwerke sehr zurück mit Spielen und kreativen Übungen zum Genus.

Es gibt kaum explizite Genusübungen wie:

(22) Mit der Wortliste [...] arbeiten. Zwölf Nomen von Seite 8 bis 15. Finden Sie die Artikel in der Liste auf Seite 231.

Name Foto Pilot $\quad$ Familie $\quad[\ldots]$

(SD: 32)

Der frequenteste Übungstyp, bei dem es um Genus geht, ist die Einsetzübung, wo fehlende Artikelformen in Sätzen oder Minitexten ergänzt werden sollen. Allerdings dürfte es sich dabei nicht so sehr um eine grammatisch-lexikalische Übung zur Genusetablierung handeln, sondern eher zu den Formen von Definit- und Indefinitartikeln bzw. Personalpronomen und zur Textreferenz durch Genuskongruenz:

(23) Das ist Handy von NETTY. Handy kostet $€ 195$. Sofa ist bequem. kostet nur $€ 299$.

Je weiter man im Lehrbuch voranschreitet, desto weniger geht es bei diesem Übungstyp um Genus, sondern vielmehr um die durch Flexionsendungen markierten Komponenten der Nominalphrase und ihre syntaktischen Funktionen wie Subjekt, Objekt usw. (soggetto, complemento oggetto, complemento di termine etc., wie es in der italienischen Schulgrammatik heißt):

(25) Completa [Ergänze] [...].

Siehst du d..... Foto? Hier ist d..... Marktplatz und da hinten ist d..... Marienkirche. Siehst du d...... Marktplatz? [...]

(Ba: AB63)

Ein weiterer Übungstyp zur Wortschatzarbeit ist die Zuordnung von Wörtern (z. B. italienisches Wort zu deutschem Pendant, Wort zu passendem Bild u. ä.). Hier laden einige Lehrwerke immer wieder implizit zum Mitlernen des Artikels ein: 
(26) Wie heißt das auf Deutsch? Abbina. [Ordne zu.]

\begin{tabular}{|c|c|}
\hline 1. porto & a. die Fußgängerzone \\
\hline 2. lago & b. das Denkmal \\
\hline 3. $[\ldots]$ & c. $[\ldots]$ \\
\hline
\end{tabular}

(Di: AB52)

(27) Lexik. Inserisci le parole nella tabella. [Schreibe die Wörter in die Tabelle.] das Schuhgeschäft - das Eiscafé - die Apotheke - [...]

\begin{tabular}{|c|c|}
\hline Kaufen & Essen und trinken \\
\hline … & (n) \\
\hline
\end{tabular}

(Ba: AB48)

Es werden vereinzelt auch unkonventionellere Wortschatzübungen angeboten, die sich ebenfalls durch einen konsequenten Miteinbezug des Genus auszeichnen wie:

(28) Cerca l'intruso. [Suche den Eindringling.]

1 der Kiosk - der Rucksack - die Tasche - das Gepäck

2 der Bahnhof - das Gleis - der Junge - der Zug

$3 \operatorname{der}[\ldots]$

(Ba: AB113)

Wortschatzübungen wie die gerade vorgestellten, die Substantive mit dem dazugehörigen Artikel präsentieren und so das Genus als "inhärente Eigenschaft von Nomen" (Bußmann 2008: 227) darstellen, sind zweifelsohne als best practice zu bewerten, um durch kontinuierliche Imitation eine korrekte Genusetablierung zu erleichtern. Leider geben einige Lehrwerke in ihren Übungen keinen Genusmarker an und berücksichtigen so diese Eigenschaft überhaupt nicht:

(29) $[\ldots]$ und verbinde.

\begin{tabular}{|c|c|}
\hline [...] 5. Sahne & [...] e. arrosto di maiale \\
\hline 6. Gemüsesuppe & f. cibo \\
\hline 7. [...] & g. [...] \\
\hline
\end{tabular}

(WB: AB120)

Das Einschleifen von Definitartikel + Substantiv im Nominativ zur Speicherung im mentalen Lexikon ist ein erfahrungsbewährtes Muster beim Genuserwerb im DaF-Unterricht. Wenn der Lerner - was ja laufend passiert - bei der Sprachproduktion das Genus eines Substantivs selegieren muss, hat er so jederzeit einen Genusabruf-Test bereit (auch wenn man einen Muttersprachler fragt, welches Genus z. B. Kosovo hat, wird er spontan so einen Test durchführen und sich fragen, ob der Kosovo, das Kosovo oder die Kosovo am besten klingt).

Im Unterricht werden Grammatikphänomene in Übungsphasen trainiert, aber auch in kognitiven Lernphasen behandelt. Lerner wollen Regeln, und Regeln bestätigen oft intuitiv Erkanntes: Sie geben den Lernern die Sicherheit, etwas richtig erfasst zu haben, und fördern das Sprachbewusstsein. Statt Regeln vorzugeben, ist es allerdings effizienter, diese die Lerner selbst entdecken zu lassen, so wie in Ba: 39:

(30) Alles klar? [...] Rileggi le parole dell'esercizio 1 e indica l'opzione giusta.

[ ] Persone e cose [ ] Solo le persone [] Solo le cose 
hanno un loro genere.

Il genere dei sostantivi tedeschi e dei loro corrispettivi italiani

[ ] corrisponde sempre. [ ] non corrisponde mai. [ ] spesso non corrisponde.

[Lies die Wörter aus der Übung 1 noch einmal und kreuz die richtige Lösung an.

[ ] Personen und Sachen [ ] Nur Personen [ ] Nur Sachen

haben ein Genus.

Das Genus der deutschen Substantive und ihrer italienischen Entsprechungen

[ ] stimmt immer überein. [ ] stimmt nie überein. [ ] stimmt oft nicht überein.] ${ }^{25}$

Solche Übungen zum entdeckenden Lernen kann man sich auch zu den Genuszuweisungsregeln vorstellen, sie fehlen aber in allen Lehrwerken.

\section{$6 \quad$ Fazit}

Die Untersuchung von an italienischen Schulen und Universitäten gängigen Lehrwerken und didaktischen Grammatiken hat gezeigt, dass zumeist eine optimale Vermittlung des Konzepts Genus aufgrund einer fehlenden Thematisierung des grammatischen Phänomens Genus, einer fehlenden Erläuterung seiner Funktion und einer fehlenden Darstellung seiner Regelhaftigkeit misslingt. Zu diesem Ergebnis kommt auch schon Wegera (1996: 4) bei seiner Untersuchung von Unterrichtsmaterialien; auch er nennt die grammatische Darstellung des Genus "zu spärlich", "wenig adäquat" und "in fast allen Fällen unzureichend".

Sicherlich hat Diehl (2000: 11) recht, wenn sie sagt:

Wer mit dem Fremdsprachenlernen Mühe hat, macht seine Unkenntnis der Grammatikregeln dafür verantwortlich; Schüler und Schülerinnen verlangen nach Grammatikunterricht, und Fremdsprachenlehrer halten es für ihre Pflicht, dieses Verlangen möglichst kompetent zu befriedigen.

Deshalb muss man daraus den Schluss ziehen, dass es angebracht wäre, wenn Deutschlerner (aber auch Deutschlehrer) über mehr Genuswissen verfügen, um die komplexe Kategorie Genus besser zu verstehen (und vermitteln zu können). Das impliziert, dass in die Lehrwerke auch mehr Genuszuweisungsregeln gehören, damit die Lerner "dem Genus nicht mehr völlig machtlos" gegenüber stehen (Meinert 1989: 21). Dass in den Deutschunterricht nur die wirklich validen und grundwortschatzbezogenen Regeln und Tendenzen gehören, um den Lerner nicht kognitiv zu überlasten, dürfte sich dabei von selbst verstehen.

Aber nicht nur die theoretische Darstellung von Genus ist verbesserungswürdig, auch das Übungsangebot lässt zu wünschen übrig: Es gibt zu wenig Übungen, die dem Lerner bei der mentalen Genusetablierung helfen könnten. Lehrwerke sollten nicht nur ein möglichst vielfältiges Angebot an Übungen enthalten, sondern auch viel mehr darauf achten, Genus bei der Wortschatzarbeit nicht zu vergessen.

Die Tradition, den Lerner Substantive immer zusammen mit dem Definitartikel als Genusmarker mitlernen zu lassen, kann nach wie vor beibehalten werden. Diese mnemotechnische Speicherleistung des Lernenden kann allerdings durch die Kombination mit Genusregeln entlastet werden (vgl. Wegera 1996: 7).

Das hier Gesagte bedeutet nicht, dass Genus mehr Platz in den Lehrwerken einzuräumen ist, als ihm bisher zusteht. Das Desiderat an die Lehrwerke ist nicht quantitativer, sondern qualitativer Art. Das Phänomen Genus sollte einfach anders angefasst und präsentiert werden, um es lernbarer (und lehrbarer) zu machen.

\footnotetext{
${ }^{25}$ Es müsste eigentlich richtig heißen: "Bezeichnungen für Personen und Sachen [...] haben ein Genus" (vgl. auch Beispiel 4 "Bezeichnungen für Obst haben immer den Artikel die" statt "Obst hat immer den Artikel die"). Diese längeren Formen, die unbekannten Wortschatz (Bezeichnungen) enthalten, sind allerdings für einen Lerner zweifelsohne schwieriger zu verstehen und zu assimilieren als die in den Lehrwerken verwendete Metonymie.
} 


\section{$7 \quad$ Anhang 1: Genusregeln auf A-Niveau}

Hier geht es nur um native Substantive und Affixe sowie um Nicht-Komposita.

Zu den Fremdwörtern sei hier nur gesagt, dass sie ihr Genus oft nach dem "Entlehnungsprinzip" (Köpcke/Zubin 1984: 89) aus der Ausgangssprache übernehmen (ital. la pizza = Femininum $\rightarrow$ die Pizza). Das gilt auch für nicht native Suffixe (italienische Nomina auf -zione, -tà etc. sind feminin, deutsche Pendants auf -tion, -tät ebenfalls). Maskulina in Sprachen, die kein Neutrum haben, können im Deutschen sowohl maskulin als neutral sein (ital. il palazzo $\rightarrow$ der Palazzo, il conto $\rightarrow$ das Konto), wenn nicht andere Regeln stärker sind, wie z. B. die SchwaRegel (ital. il garage $=$ Maskulinum, aber die Garage $=$ Femininum).

\subsection{Morphologische Regeln}

\subsubsection{Wortbildungsregeln}

\subsubsection{Derivationsregeln (1): Produktive Affixe}

\begin{tabular}{|c|c|}
\hline M1a & $\begin{array}{l}\text { Denominale Nomina agentis, die durch Anhängen von -er gebildet werden, sind immer } \\
\text { maskulin (Eisenbahn } \rightarrow \text { der Eisenbahner); hierzu zählen auch Bewohnerbezeichnungen auf } \\
\text {-er }(\text { Hamburg } \rightarrow \text { der Hamburger) sowie Nomina agentis auf die weniger produktiven und } \\
\text { frequenten Suffixe -ler, -ner, -iker ( Sport } \rightarrow \text { der Sportler, Rente } \rightarrow \text { der Rentner, Alkohol } \rightarrow \\
\text { der Alkoholiker). }\end{array}$ \\
\hline M1b & $\begin{array}{l}\text { Deverbative Nomina agentis, die durch Anhängen von -er gebildet werden, sind immer } \\
\text { maskulin (arbeiten } \rightarrow \text { der Arbeiter). }\end{array}$ \\
\hline M1c & $\begin{array}{l}\text { Auch andere Deverbativa, die durch Anhängen von -er gebildet werden, sind immer masku- } \\
\text { lin wie } \\
\text { - Gerätebezeichnungen (fernsehen } \rightarrow \text { der Fernseher }) \text {; } \\
\text { - Vorgangs- und Geschehensbezeichnungen }(\text { fehlen } \rightarrow \text { der Fehler }) \text {. }\end{array}$ \\
\hline M1d & $\begin{array}{l}\text { Derivate von Numeralia auf -er sind immer maskulin }(50 \rightarrow \text { der Fünfziger }) \text {, auf }-(s) \text { tel neu- } \\
\text { tral }(4 \rightarrow \text { das Viertel, } 20 \rightarrow \text { das Zwanzigstel }) \text {. }\end{array}$ \\
\hline M2 & $\begin{array}{l}\text { Denominale Nomina agentis, die durch Anhängen von -in gebildet werden, sind immer fe- } \\
\text { minin (Lehrer } \rightarrow \text { die Lehrerin). }\end{array}$ \\
\hline M3 & $\begin{array}{l}\text { Denominale Diminutiva, die auf die Wortbildungssuffixe -chen und -lein enden, sind immer } \\
\text { neutral (Haus } \rightarrow \text { das Häuschen, das Häuslein). }\end{array}$ \\
\hline M4 & $\begin{array}{l}\text { Substantive, die auf die Wortbildungssuffixe -ung, -schaft, -heit, -keit, -ei enden, sind immer } \\
\text { feminin (üben } \rightarrow \text { die Übung, wissen } \rightarrow \text { die Wissenschaft, krank } \rightarrow \text { die Krankheit, schwierig } \\
\rightarrow \text { die Schwierigkeit, Bäcker } \rightarrow \text { die Bäckerei). }\end{array}$ \\
\hline M5 & Substantive aus $G e-+$ Verbstamm + -e (fragen $\rightarrow$ das Gefrage $)$ sind immer neutral. \\
\hline
\end{tabular}

zu M1c: Diese Deverbativa werden jedoch nicht unbedingt von Lernern als solche erkannt, da sie oft vor den Verben gelernt werden (schlüpfen $\rightarrow$ der Schlüpfer) oder können sogar von Muttersprachlern nicht immer als solche identifiziert werden (walzen $\rightarrow$ der Walzer). Daher dürfte diese Regel nur bedingt auf A-Niveau von Nutzen sein.

zu M5: Dieses Wortbildungsmuster ist hochproduktiv. Es wundert daher, dass es nicht einmal in den didaktischen Grammatiken erwähnt wird. Dabei wären doch Äußerungen wie Hör auf mit dem Gefrage/Getanze/Getue etc. auch auf A-Niveau durchaus denkbar. Vielleicht liegt es daran, dass einige (allerdings wenige) Verben auszuschließen sind (reflexive Verben, Modalverben, Verben auf -ieren und Verben mit untrennbarem Präfix). Mehr dazu bei Fleischer (1983: 185-187).

Bei den Suffixen handelt es sich im Prinzip um das "Letzt-Glied-Prinzip": Substantive, die aus zwei oder mehr Wörtern zusammengesetzt sind, wobei das letzte Element ein Substantiv 
ist, übernehmen das Genus des letzten Elements wie die Stadt $\rightarrow$ die Altstadt, der Tisch $\rightarrow$ der Schreibtisch, das Zimmer $\rightarrow$ das Kinderzimmer etc. (vgl. Köpcke/Zubin 1984: 89).

\subsubsection{Konversionsregeln: Produktive Konversion}

M6 Substantivierte Infinitive - wie auch andere Substantivierungen durch Übergänge aus anderen Wortklassen ${ }^{26}$ - sind neutral (leben $\rightarrow$ das Leben, essen $\rightarrow$ das Essen). ${ }^{27}$

\subsubsection{Derivationsregeln (2): Improduktive oder nicht frequente Präfixe und Suffixe}

\subsubsection{1 "Pseudosuffixe"}

Unter Pseudosuffixen versteht man "Wortausgänge [...], die nicht den morphologischen Status von Derivationssuffixen haben, aber auch nicht zum Stamm - als Basis für Wortbildungsprozesse - gehören" (Hoberg 2004: 91). Dazu sei anzumerken, dass -e, -er etc. auch den Status von Derivationssuffixen haben können (reden $\rightarrow$ die Rede, flach $\rightarrow$ die Fläche, schalten $\rightarrow$ der Schalter), ihn aber nicht immer haben (wie in die Katze, der Vater) - s. dazu Fleischer 1983: 133f. Diese Unterscheidung dürfte aber jeden Deutschlerner überfordern. $\mathrm{Zu}$ den Pseudosuffixen werden meist auch -em und -(e)s gezählt; im Grundwortschatz gibt es allerdings kaum Wörter mit diesem Wortausgang; sie sind meist maskulin (der Atem, der Schnaps).

Es ist zu erwägen, ob es didaktisch sinnvoll ist, die -e-, -en-, -er-, -el-Regeln als phonologische Regeln zu betrachten.

\begin{tabular}{|c|c|}
\hline M7 & Substantive auf - $e$ sind fast immer feminin (die Blume) - sog. Schwa-Regel. \\
\hline M8 & Substantive auf -en sind fast immer maskulin (der Wagen). \\
\hline M9 & Substantive auf -er sind meist maskulin (der Teller). \\
\hline M10 & $\begin{array}{l}\text { Substantive auf -el sind oft maskulin (der Apfel), zu einem Viertel allerdings feminin (die } \\
\text { Tafel), selten neutral (das Rätsel }{ }^{28} \text { ). }\end{array}$ \\
\hline
\end{tabular}

zu M7: Substantive mit Derivationssuffix $-e$ sind immer feminin (reden $\rightarrow$ die Rede, flach $\rightarrow$ die Fläche), andere Substantive auf -e sind es fast immer. Wenn man die in der Regelhierarchie höher stehenden Regeln S1a (Sexus wie der Junge) oder S1b (generisches Maskulinum wie der Kunde) anwendet, die das Genus der Maskulina erklären, die allesamt zur Deklinationsklasse mit Akkusativ, Dativ und Genitiv auf -(e)n gehören - s. dazu Regel M16 -, bleiben nach Durrell (1992: 151) nur wenige Ausnahmen von der Schwa-Regel:

- die acht Maskulina auf -e, die die kleine Deklinationsklasse mit Dativ und Akkusativ auf - $n$ und Genitiv auf - $n s$ bilden wie der Name (s. Regel M16);

- acht weitere, nämlich der Käse als einziges Maskulinum und die Neutra das Ende, das Auge, das Interesse sowie die auf A-Niveau nicht relevanten das Erbe sowie das Image, das Prestige, das Regime (allerdings ohne Schwa-Auslaut).

Dabei vergisst Durrell in seiner Liste allerdings eine Reihe von Substantiven mit dem Präfix Ge- bzw. Zirkumfix Ge-...e (das Gefrage, das Gebirge, das Gebäude, das Gemälde; der Ge-

\footnotetext{
${ }^{26}$ wie $j a \rightarrow$ das $J a, i c h \rightarrow$ das Ich etc. Diese Regeln sind jedoch auf A-Niveau nicht relevant. Ob es sich bei alt $\rightarrow$ der Alte, die Alte, das Alte um substantivierte Adjektive (so die meisten Lehrwerke und Grammatiken) oder um substantivischem Gebrauch der Adjektive (so Helbig/Buscha 2001: 223f.) handelt, sei dahingestellt. Auf jeden Fall spielt dieses Konversionsmuster auf A-Niveau keine Rolle.

${ }^{27}$ S. dazu auch Regel M8.

${ }^{28}$ sowie Substantivierungen wie das Viertel, die unter die in der Regelhierarchie höher stehenden Derivationsregel M1d fallen.
} 
fährte) - s. dazu auch Regel M5 und M11. Trotzdem bleibt die Liste dieser Substantive so klein, dass sie einfach als Ausnahmen von dieser sog. Schwa-Regel lernbar sind.

zu M8: Wenn man die in der Regelhierarchie höher stehenden Regeln wie M3 (das Häuschen) oder M6 (das Essen) anwendet, bleibt eine kleine Anzahl an Ausnahmen auf A-Niveau wie das Zeichen, das Waschbecken, das Kissen. Himmelsrichtungen enden auf -en und sind immer maskulin (der Norden) und fallen unter diese Regel (es bedarf also keiner speziellen Regel für diese vier Wörter, wie sie des öfteren formuliert wird). Auch der Gefallen und der Schaden gehören hierhin, wobei es nahe liegt, dass ein DaF-Lerner sie fälschlicherweise als substantivierte Infinitive ansieht und M6 zur Anwendung bringt.

zu M9: Die meisten Substantive auf -er sind maskulin aufgrund von M1. Einige Verwandtschaftsbezeichnungen enden auf -er und sind aufgrund von S1a entweder maskulin (der Vater, der Bruder) oder feminin (die Mutter, die Tochter). Es bleibt nur eine recht kleine Gruppe übrig: Diese Substantive sind fast nie feminin (die Butter), sondern maskulin (der Teller, der Hunger) oder neutral (das Fenster, das Fieber). Weiter zu spezifizieren, wie es Harnisch (2001) tut, dürfte allerdings zumindest den schulischen DaF-Unterricht überfordern (Substantive auf -er, die Gefäße und Instrumente beschreiben, sind maskulin, die Material und Gebäudeteile beschreiben, sind neutral etc.). Zur Semantik der deutschen Affixe s. auch Donalies (2002: 105f.).

\subsection{Weitere Affixe}

\begin{tabular}{|l|l|}
\hline M11 & Substantive mit dem Präfix Ge- sind fast immer neutral (schenken $\rightarrow$ das Geschenk). \\
\hline M12 & Substantive auf -ling sind fast immer maskulin $($ früh $\rightarrow$ der Frühling). \\
\hline M13 & $\begin{array}{l}\text { Substantive auf -nis und -tum sind meist neutral (ergeben } \rightarrow \text { das Ergebnis, eigen } \rightarrow \text { das } \\
\text { Eigentum). }\end{array}$ \\
\hline M14 & Substantive aus Verbstamm + -t sind meist feminin (fahren $\rightarrow$ die Fahrt). \\
\hline M15 & $\begin{array}{l}\text { Substantive aus Verbstamm + Nullallomorph (Ø) sind meist maskulin }(\text { fallen } \rightarrow \text { der Fall, } \\
\text { küssen } \rightarrow \text { der Kuss), aber oft auch Neutrum (zelten } \rightarrow \text { das Zelt, spielen } \rightarrow \text { das Spiel), fast } \\
\text { nie Femininum (wählen } \rightarrow \text { die Wahl, lüften } \rightarrow \text { die Luft }) .\end{array}$ \\
\hline
\end{tabular}

zu M11: Nur Ge- kann als einziges Präfix das Genus determinieren, alle anderen Präfixe haben keinen Einfluss darauf (der Schluss, der Entschluss, der Anschluss usw.).

Neutral sind die meisten Deverbativa mit dem Präfix $G e-$, unabhängig davon, ob sie auf - $e$ enden oder nicht (schenken $\rightarrow$ das Geschenk, trinken $\rightarrow$ das Getränk, auch wiegen $\rightarrow$ das Gewicht, bauen $\rightarrow$ das Gebäude, malen $\rightarrow$ das Gemälde - s. auch M5) sowie die meisten denominalen Substantive mit Ge- (Berge $\rightarrow$ das Gebirge, Mus $\rightarrow$ das Gemüse), wobei aber ein Lerner aufgrund seines reduzierten Wortschatzes nicht unbedingt die verbale bzw. substantivische Basis identifizieren kann.

Ausnahmen von M11 sind: der Gesang, der Geruch, der Gedanke, die Geburt, die Geschichte u. a.

zu M12: Substantive auf -ling sind immer maskulin, es sei denn, das -l- gehört nicht zum Suffix (wie in die Reling).

zu M14: Allerdings ist der Verbstamm oft nicht klar erkennbar (ankommen $\rightarrow$ die Ankunft, gebären $\rightarrow$ die Geburt, schreiben $\rightarrow$ die Schrift, sehen $\rightarrow$ die Sicht); außerdem gibt es Ausnahmen (das Gesicht).

zu M15: Dieses Wortbildungsmuster wird z. T. auch als "Konversion aus Verbwurzeln" angesehen, wobei von der Wurzel ausgegangen wird, "die endungslos sonst nur im Imperativ 
auftritt"29 (Hentschel/Weydt 2003: 193). Problematisch ist es manchmal, die Derivationsrichtung zu bestimmen (lüften $\rightarrow$ Luft oder eher Luft $\rightarrow$ lüften?).

\subsubsection{Flexionsregeln}

\subsubsection{Genitivregeln}

\begin{tabular}{|c|c|}
\hline M16 & $\begin{array}{l}\text { - Genitivendung nein } \rightarrow \mathrm{F}(\text { die Uhr mit Genitiv Uhr), Genitivendung ja } \rightarrow \mathrm{M} / \mathrm{N} \text { (der Lehrer } \\
\text { mit Genitiv Lehrers, das Kind mit Genitiv Kindes) } \\
\text { - Genitiv auf - }(e) n \text { oder -en }(s) \rightarrow \mathrm{M}(\text { der Herr mit Genitiv Herrn bzw. der Name mit Genitiv } \\
\text { Namens) } \\
\text { - Genitiv auf }-(e) s \rightarrow \mathrm{M} / \mathrm{N}(\text { der Arzt } \text { mit Genitiv Arztes bzw. das Ei mit Genitiv Eis) }\end{array}$ \\
\hline
\end{tabular}

zu M16:

Genitiv auf -(e)n: Die Nomina mit Genitiv auf -en haben alle auch im Akkusativ und Dativ -en. Diese Deklinationsklasse macht $10 \%$ der maskulinen Substantive aus. Hier finden sich fast nur Personenbezeichnungen; Bezeichnungen für Nicht-Personen sind auf A-Niveau nicht relevant (der Affe, der Löwe).

Genitiv auf -en(s): Durrell (1992: 151) zählt acht Substantive, die diese Deklinationsklasse bilden: der Name, der Buchstabe, der Gedanke, der Glaube, der Wille und der Friede(n), der Funke(n), der Same(n) mit fakultativem finalen - $n$. Andere Grammatiken (z. B. Duden) erweitern diese Liste mit weiteren Substantiven auf $-e(n)$, die jedoch allesamt auf A-Niveau unwichtig sind. Die zu dieser kleinen Deklinationsklasse gehörenden Substantive enden alle auf $-e(n)$ und sind alle maskulin bis auf das Herz.

\subsubsection{Pluralregeln}

\begin{tabular}{|l|l|}
\hline M17 & - Plural auf $-\varnothing \rightarrow$ nicht $\mathrm{F}($ Lehrer $\rightarrow$ der Lehrer, Fenster $\rightarrow$ das Fenster $)$ \\
& - Plural auf $-(e) n \rightarrow$ wahrscheinlich F $($ Uhren $\rightarrow$ die Uhr, Kartoffeln $\rightarrow$ die Kartoffel $)$ \\
& - Plural auf $-e \rightarrow$ wahrscheinlich $\mathrm{M}$, eventuell auch $\mathrm{N}($ Bleistifte $\rightarrow$ der Bleistift $)$ \\
& - Plural auf $-e r \rightarrow$ wahrscheinlich N $($ Bücher $\rightarrow$ das Buch $)$ \\
& - Plural auf $-s \rightarrow \mathrm{M}, \mathrm{N}, \mathrm{F}$
\end{tabular}

\section{zu M17:}

Plural auf -Ø: Es handelt sich in dieser Klasse nur um nicht-feminine morphologisch markierte Substantive auf -er, -el, -en, -chen, -lein, -tel. Die femininen Substantive auf -er, -el, -en bilden den Plural auf $-n$.

Plural auf -(e)n: Laut Augst (1975) bilden 75\% der Feminina den Plural auf -(e)n (die restlichen $25 \%$ bilden ihn auf $-e$ ). In diese Gruppe gehören auch alle Substantive, die im Genitiv -(e)n (der Herr mit Genitiv Herrn) oder -ens haben (der Name mit Genitiv Namens) - s. Regel M16 -, kaum andere Maskulina und kaum Neutra.

Plural auf $-e$ : Auch wenn $75 \%$ der Neutra ihren Plural auf $-e$ bilden (die restlichen $25 \%$ bilden ihn auf -er, fast keine auf -en), ist die Wahrscheinlichkeit, dass es sich um ein Maskulinum handelt, einfach größer, weil fast alle Maskulina diesem Pluraltyp folgen (nur wenige bilden den Plural auf -en oder -er) und es einfach viel mehr Maskulina gibt als Neutra. Laut Augst (1975: 37f.) sind 67\% der Substantive Maskulina und nur 20\% Neutra. Andere Zahlen finden sich bei Hoberg (2004: 81-84): Für sie hängt die Genusverteilung einerseits von der Sprachvarietät zusammen (gesprochene Alltagsprache: hohe M-Werte bis zu 45\%, Zeitungs-

\footnotetext{
${ }^{29}$ Es soll hier aber nicht weiter auf die Problematik der Bildung von Imperativformen eingegangen werden.
} 
sprache: hohe F-Werte bis zu über 50\%) und andererseits von der Zahl der Substantive, die ins Spiel kommen (je mehr Substantive, desto weniger N).

Plural auf -s: Für Gaeta (2007: 71) ist $-s$-Plural Default-Plural, da er nur von Substantiven gebildet wird, die untypisch für das Deutsche sind (Substantive auf $-a,-i,-o,-u$, Abkürzungen, Fremdwörter).

\subsection{Semantische Regeln}

\begin{tabular}{|l|l|}
\hline S1a & $\begin{array}{l}\text { Sexus: Männliche Personen sind praktisch immer maskulin, weibliche Personen feminin } \\
\text { (Der Vater, die Mutter, der Onkel und die Tante. Lukas ist ein guter Lehrer, Anna eine gute } \\
\text { Lehrerin). }\end{array}$ \\
\hline S1b & $\begin{array}{l}\text { Generisches Maskulinum: Maskulin ist auch eine Art "Default-Genus" für Personen- } \\
\text { bezeichnungen (Hentschel/Weydt 2003: 162) (Ein Lehrer hat es schwer.). }\end{array}$ \\
\hline S2 & $\begin{array}{l}\text { Gruppenbedeutungssprinzip } \\
\text { a) wie: }{ }^{31} \\
\text { woch, der Januar, der Sommer, aber die Nacht); } \\
\text { b) Niederschläge sind fast immer maskulin (der Schnee); } \\
\text { c) Alkoholika sind fast immer maskulin (der Wein, aber das Bier). }\end{array}$ \\
\hline S3 & $\begin{array}{l}\text { "Unterklassifizierungsprinzip" (Köpcke/Zubin 1984: } 89): 32 \text { Ein Substantiv oder Name über- } \\
\text { nimmt das Genus des "Leitwortes" (Di Meola 2007b: } 90) \text { wie der Alkohol } \rightarrow \text { der Wein, der } \\
\text { Whisky; der Monat } \rightarrow \text { der Januar, der Februar; die Zahl } \rightarrow \text { die Eins, die Zwei. }\end{array}$ \\
\hline S4 & $\begin{array}{l}\text { Vollformprinzip: Kurzformen und Akronyme erhalten ihr Genus von der "Vollform" (Fi- } \\
\text { scher 2005: } \\
\rightarrow \text { das Alt } \text { ) } \text { wie die Lokomotive } \rightarrow \text { die Lok, das Atomkraftwerk } \rightarrow \text { das AKW, das Altbier }\end{array}$ \\
\hline S5 & $\begin{array}{l}\text { "Bedeutungsprinzip" (ibd:: 90): Neue Wörter erhalten ihr Genus von Wörtern mit ähnlicher } \\
\text { Bedeutung wie das Telefon } \rightarrow \text { das Handy, das Bild } \rightarrow \text { das Foto. }\end{array}$ \\
\hline
\end{tabular}

zu S1a: Die Sexus-Regel weist wenige Ausnahmen auf, die auf A-Niveau jedoch keine Rolle spielen (das Weib). Auf diesem Niveau relevante Ausnahmen lassen sich mit den Regeln M1-3 erklären, die in der Hierarchie über den Sexus-Regeln stehen (Familie Meier hat einen neuen Babysitter: Anna [...]. $\rightarrow$ Regel M1b; Das Mädchen heißt Anna. $\rightarrow$ M3), bei Fremdwörtern mit dem Ursprungsgenus (Herr Meier ist eine interessante Person $\rightarrow$ Person $=\mathrm{Fe}-$ mininum wie lat. persona).

Von Sexus-Regel kann man auch bei Tieren sprechen (sehr detailliert dazu Hentschel/Weydt 2003: 160-162), was allerdings auf A-Niveau nicht interessant ist (man denke an der Hund, die Katze, das Pferd).

zu S1b: Formen wie (der) Lehrer drücken nicht unbedingt Sexus ausdrücken. In Lehrer haben es schwer/Ein Lehrer hat es schwer sind auch Frauen gemeint. - Zum kontroversen Thema generisches Maskulinum: s. o.

zu S3-5: Allerdings gibt es nicht wenige Ausnahmen: das Motorrad $\rightarrow$ die BMW, die Fotografie $\rightarrow$ das Foto, der Brief $\rightarrow$ die Mail.

\footnotetext{
${ }^{30}$ Engel (2004: 227) spricht von Gruppenbedeutung.

${ }^{31}$ Die Hauptregeln sind in jeder didaktischen und wissenschaftlichen Grammatik nachzulesen, wobei es allerdings keine zahlenmäßige und inhaltliche Übereinstimmung zwischen den verschiedenen Handbüchern gibt. Aufgrund ihrer didaktischen Irrelevanz werden hier keine weiteren semantischen Ansätze wie die Köpcke/Zubin (1983 etc.) aufgegriffen.

${ }^{32}$ Noch detaillierter bei Fischer (2005: 90-97): Bedeutungsprinzip, Gattungsprinzip, Metapher- und Metonymieregel, Oberbegrifftendenz.
} 


\subsection{Phonologische Regeln}

\begin{tabular}{|c|c|}
\hline $\mathrm{Ph} 1$ & $\begin{array}{l}\text { Substantive auf -ich und -ig sind fast immer maskulin (der Teppich, der Essig), auf - } t \text { ten- } \\
\text { denziell feminin (die Arbeit). }\end{array}$ \\
\hline $\mathrm{Ph} 2$ & $\begin{array}{l}\text { Je mehr Konsonanten am Wortanfang oder am Wortende sind, desto wahrscheinlicher ist es, } \\
\text { dass das Substantiv maskulin ist (der Strumpf). } \\
\text { Ausnahmen: Substantive auf -[t]. Tendenziell gilt: } \\
\text { - auf -[عt] neutral (das Bett); } \\
\text { - auf (Konsonant }+)[\mathrm{f} / \mathrm{c} / \mathrm{x}]+t \text { feminin (die Kraft). }\end{array}$ \\
\hline $\mathrm{Ph} 3$ & Einsilber sind oft maskulin ( er Arm) \\
\hline
\end{tabular}

zu Ph1: -ich und -ig sind weder Suffixe noch Pseudosuffixe; für Eisenberg (2006a: 427) handelt es sich bei den Beispielen um "morphologisch einfache Wörter", die nicht weiter zerlegt werden können. Substantive auf -ich sind immer maskulin, auch Personenbezeichnungen, die auf das Suffix -rich enden (der Wüterich wegen S1b). Bei den Substantiven auf -ig gibt es rare Ausnahmen (die Hallig).

zu Ph2: Laut Zubin/Köpcke (1981: 441) ergibt sich eine sehr hohe Validität dieser Regel (Zahlen z. B. auch bei Eisenberg 2006b: 153), die jedoch mehrfach angefochten wird (wie von Fischer 2005: 214 oder Feigs 2007: 49). An anderen Stellen spezifizieren Zubin/Köpcke noch detaillierter als hier (Anlaut [kn]-, [d/t] $+r$ - etc.) wie in Köpcke/Zubin (1983 usw.).

zu Ph3: Diese Tendenz (von Regel kann man hier sicherlich nicht mehr sprechen) impliziert auf den ersten Blick eine sehr hohe Fehlerquote von einem Drittel, denn fast ein Viertel der Einsilber sind schließlich Neutra (das Buch) und knapp 15\% Feminina (die Uhr). ${ }^{33}$ Die Fehlerquote sinkt allerdings merklich bei Anwendung aller vorher genannten Regeln.

\section{$8 \quad$ Anhang 2: Untersuchte Lehrwerke und didaktische Grammatiken (mit Auflösung der Siglen)}

\subsection{Liste der untersuchten Lehrwerke}

\subsubsection{In Italien veröffentlichte Lehrwerke}

$\mathrm{Ba}=\quad$ Weerning, Marion (2007): Basis Deutsch. Lehrwerk für Deutsch als Fremdsprache. Kursbuch und Arbeitsbuch. Al+. Milano/Ismaning: Edumond Le Monnier/Hueber.

$\mathrm{Di}=\quad$ Motta, Giorgio (2004): Direkt 1. Ein Lehrwerk für Deutsch als Fremdsprache. Kursbuch und Arbeitsbuch. Torino: Loescher.

$\mathrm{Ga}=\quad$ Quartapelle, Franca (2005): Galaktisch. Ein deutsches Lernbuch für interkulturelles Handeln. Basisbuch 1. Arbeitsbuch 1. Milano: Zanichelli.

$\mathrm{St}=\quad$ Vanni, Angela/Delor, Regine (2010): Stimmt! 1. Deutsche Sprache und Kultur für junge Leute. Kursbuch + Arbeitsbuch. Milano/Torino: Pearson Italia (Edizioni Lang).

VD-AB = Curci, Anna Maria/Roth, Susanne M./Bente Pieper, Siri (2005b): Vitamin D 1. Arbeitsbuch . Firenze: Le Monnier.

VD-L = Curci, Anna Maria/Roth, Susanne M./Bente Pieper, Siri (2005a): Vitamin D 1. Ein modulares Lehrwerk für Deutsch. Firenze: Le Monnier.

$\mathrm{WB}=$ Catani, Cesarina/Greiner, Herbert/Pedrelli, Elena (2006): Wie bitte? Kompakt. Ein Lehrwerk für deutsche Sprache und Kultur. Kursbuch $1+$ Arbeitsbuch 1. Neue Ausgabe. Milano: Zanichelli.

\footnotetext{
${ }^{33}$ Laut Köpcke (1982: 45) sind 64\% M, 14\% F, 22\% N.
} 


\subsubsection{In Deutschland veröffentlichte Lehrwerke}

$\mathrm{BP}=\quad$ Lemcke, Christiane/Rohrmann, Lutz/Scherling, Theo (2002): Berliner Platz 1. Deutsch im Alltag für Erwachsene. Berlin etc.: Langenscheidt.

$\mathrm{Eu}=\quad$ Funk, Hermann/Koenig, Michael (eds.) (2005): Eurolingua Deutsch 1. Teilband 1. Neue Ausgabe. Berlin: Cornelsen.

Op-AB = Müller, Martin et al. (2004): Optimal Al. Lehrwerk für Deutsch als Fremdsprache. Libro degli esercizi. Italienische Ausgabe des Arbeitsbuches. Berlin/München: Langenscheidt.

$\mathrm{Op}-\mathrm{L}=$ Müller, Martin et al. (2004): Optimal A1. Lehrwerk für Deutsch als Fremdsprache. Lehrbuch. Berlin/München: Langenscheidt.

$\mathrm{SD}=\quad$ Funk, Hermann/Kuhn, Christina/Demme, Silke (2005): studio d Al. Deutsch als Fremdsprache. Kurs- und Übungsbuch. Berlin: Cornelsen.

\subsection{Liste der untersuchten didaktischen Grammatiken}

\subsubsection{In Italien veröffentlichte didaktische Grammatiken}

$\mathrm{BF}=\quad$ Bruno, Elena/Franch, Raffaela (2004): Deutsche Grammatik. Grammatica di riferimento per lo studio della lingua tedesca. Torino: Capitello.

DF $=\quad$ Difino, Elisabetta/Fornaciari, Paola (1992): Deutsch klipp und klar! Ein deutsches Übungsbuch. Milano: Principato.

LP $=\quad$ Lackkamp, Monica/Portius, Helga (1995): Der treue Freund. Referenzgrammatik der deutschen Sprache. Torino: Edisco.

$\mathrm{SM}=\quad$ Seiffarth, Achim/Medaglia, Cinzia (1998): Arbeitsgrammatik. Genova: Cideb.

$\mathrm{WM}=$ Weerning, Marion/Mondello, Mariano (2004): Dies und Das Neu. Grammatica di tedesco con esercizi. Genova: Cideb.

\subsubsection{In Deutschland veröffentlichte didaktische Grammatiken}

DS $=\quad$ Dreyer, Hilke/Schmitt, Richard (1993): Grammatica tedesca con esercizi. Ismaning: Hueber. (Italienische Ausgabe von Dreyer, Hilke/Schmitt, Richard (1985): Lehr- und Übungsbuch der deutschen Grammatik. München: Verlag für Deutsch).

FD $=\quad$ Frey, Evelyn/Dittrich, Roland (2005): Sequenzen. Übungsgrammatik Deutsch als Fremdsprache. Berlin: Cornelsen.

FT $=\quad$ Fandrych, Christian/Tallowitz, Ulrike (2000): Klipp und Klar. Übungsgrammatik Grundstufe Deutsch. Stuttgart: Klett.

$\mathrm{He}=\quad$ Heringer, Hans-Jürgen (2002): Deutschexpress. Lernergrammatik Deutsch als Fremdsprache. Berlin: Cornelsen.

$\mathrm{Lu}=\quad$ Luscher, Renate (2001): Übungsgrammatik für Anfänger Deutsch als Fremdsprache. Ismaning: Hueber.

Rei $=\quad$ Reimann, Monika (1998): Grammatica di base della lingua tedesca . Con esercizi . Ismaning: Hueber. (Edizione italiana di Reimann, Monika (1996): Grundstufen-Grammatik für Deutsch als Fremdprachen. Erklärungen und Übungen. Ismaning: Hueber.)

SS = Späth, Christine/Sailer, Marion (2002): Und jetzt ihr! Basisgrammatik für Jugendliche. Ismaning: Hueber.

\section{Literatur}

Alella, Anna Maria/Marini, Ida (1991): Grammatica tedesca. Milano: Signorelli (erstmals erschienen: 1946, Torino: Società Editrice Internazionale).

Augst, Gerhard (1975): Untersuchungen zum Morpheminventar der deutschen Gegenwartssprache. Tübingen: Narr. 
Barkowski, Hans/Krumm, Hans-Jürgen (eds.) (2010): Fachlexikon Deutsch als Fremdsprache und Zweitsprache. Tübingen: Narr/Francke/Attempto.

Braun, Friederike/Sczesny, Sabine/Stahlberg, Dagmar (2002): "Das generische Maskulinum und die Alternativen. Empirische Studien zur Wirkung generischer Personenbezeichnungen im Deutschen". Germanistische Linguistik 166-182: 77-87.

Bates, Elizabeth et al. (1995): "Gender and lexical access in Italian". Perception and Psychophysics 57: 847-862.

Brugmann, Karl (1889): "Zur Frage der Entstehung des grammatischen Geschlechts. (Aus anlass von Roethe's vorwort zum neudruck des 3. bandes der Grimm'schen grammatik)". Techmers Internationale Zeitschrift für allgemeine Sprachwissenschaft 4: 100-109. Reprint in: Sieburg, Heinz (ed.) (1997): Sprache - Genus/Sexus. Frankfurt a. M. etc., Lang: 62-68.

Bußmann, Hadumod (2005): "Haben Sprachen ein Geschlecht? Genus/Gender in der Sprachwissenschaft" In: Bußmann, Hadumod/Hof, Renate (eds.): Genus. Geschlechterforschung/Gender Studies in den Kultur- und Sozialwissenschaften. Ein Handbuch. Stuttgart, Kroener: 482-519.

Bußmann, Hadumod (ed.) (2008): Lexikon der Sprachwissenschaft. Stuttgart: Kroener.

Chini, Marina (1998): "Genuserwerb des Italienischen durch deutsche Lerner". In: Wegener, Heide: Eine zweite Sprache lernen: empirische Untersuchungen zum Zweitspracherwerb. Tübingen, Narr: 39-60. (= Tübinger Beiträge zur Linguistik Series A, Language Development 24).

Christen, Helen (2000a): "Deutsch in Genf". In: Diehl, Erika et al.: Grammatikunterricht: alles für der Katz? Untersuchungen zum Zweitsprachenerwerb Deutsch. Tübingen, Niemeyer: 13-24. (= Reihe Germanistische Linguistik 220).

Christen, Helen (2000b): "Der Brot, die Mädchen, das Führerschein - Der Erwerb der deutschen Genera". In: Diehl, Erika et al.: Grammatikunterricht: alles für der Katz? Untersuchungen zum Zweitsprachenerwerb Deutsch. Tübingen, Niemeyer: 167-197. (= Reihe Germanistische Linguistik 220).

Demming, Keith M./Kemmer, Suzanne (eds.) (1990): On language: Selected writings of Joseph H. Greenberg. Stanford: Stanford University Press.

Di Meola, Claudio (2004): La linguistica tedesca. Un'introduzione con esercizi e bibliografia ragionata. Roma: Bulzoni.

Di Meola, Claudio (2007a): "Genuszuweisung im Deutschen als globaler und lokaler Strukturierfaktor des nominalen Lexikons". Deutsche Sprache 35: 138-158.

Di Meola, Claudio (2007b): "Neutrale Genuszuweisung im Deutschen". In: Di Meola, Claudio et al. (eds.): Perspektiven Zwei. Akten der 2. Tagung Deutsche Sprachwissenschaft in Italien (Rom, 9.-11. Februar 2006). Roma, Istituto di Studi Germanici: 87-99.

Diehl, Erika (2000): "Einleitung". In: Diehl, Erika et al.: Grammatikunterricht: alles für der Katz? Untersuchungen zum Zweitsprachenerwerb Deutsch. Tübingen, Niemeyer: 3-11. (= Reihe Germanistische Linguistik 220).

Donalies, Elke (2002): Die Wortbildung im Deutschen. Ein Überblick. Tübingen: Narr. (= Studien zur deutschen Sprache 27).

Duden. Die Grammatik (2006). Mannheim etc.: Duden. (= Der Duden in zwölf Bänden, Band 4).

Durrell, Martin (1992): Using German. A guide to contemporary usage. Cambridge: Cambridge University Press.

Eisenberg, Peter (2006a). Grundriss der deutschen Grammatik. Band 1: Das Wort. 3., durchgesehene Auflage. Stuttgart/Weimar: Metzler.

Eisenberg, Peter (2006b). Grundriss der deutschen Grammatik. Band 2: Der Satz. 3., durchgesehene Auflage. Stuttgart/Weimar: Metzler.

Engel, Ulrich (2004): Deutsche Grammatik. Neubearbeitung. München: Iudicium. 
Feigs, Wolfgang (2007): "Zur Genuszuweisung aufgrund phonologischer Merkmale im Deutschen". Zeitschrift für Angewandte Linguistik 46: 41-55.

Fischer, Rudolf-Josef (2005): Genuszuordnung. Theorie und Praxis am Beispiel des Deutschen. Frankfurt a. M. etc.: Lang (= Europäische Hochschulschriften 21, Linguistik 281).

Fleischer, Wolfgang (1983): Wortbildung der deutschen Gegenwartssprache. 5. Aufl. Leipzig: VEB.

Fleischer, Wolfgang/Helbig, Gerhard/Lerchner, Gotthard (eds.) (2001): Kleine Enzyklopädie Deutsche Sprache. Frankfurt a. M.: Lang.

Gaeta, Livio (2007): "Überlegungen zum deutschen Plural". In: Di Meola, Claudio et al. (eds.): Perspektiven Zwei. Akten der 2. Tagung Deutsche Sprachwissenschaft in Italien (Rom, 9.-11. Februar 2006). Roma, Istituto di Studi Germanici: 68-85.

Götze, Lutz (1984): Artikelwörter und Genusverdeutlichung in der deutschen Gegenwartssprache. München: Goethe-Institut (= Publikationen der Projektgruppe "Lehrschwierigkeiten im Fach 'Deutsch als Fremdsprache' " 7).

Grimm, Jacob (1890): Deutsche Grammatik. Bd. 3, neuer vermehrter Abdruck besorgt durch Gustav Roethe und Edward Schröder. Gütersloh: Bertelsmann.

Harnisch, Rüdiger (2001): Grundform- und Stamm-Prinzip in der Substantivmorphologie des Deutschen. Synchronische und diachronische Untersuchung eines typologischen Parameters. Heidelberg: Winter.

Helbig, Gerhard/Buscha, Joachim (2001). Deutsche Grammatik. Ein Handbuch für den Ausländerunterricht. Berlin etc.: Langenscheidt.

Hentschel, Elke/Weydt, Harald (2003). Handbuch der deutschen Grammatik (3., völlig neu bearb. Aufl.). Berlin/New York: de Gruyter.

Heupel, Carl (1973): Taschenbuch der Linguistik. München: List.

Hoberg, Ursula (2004): Grammatik des Deutschen im europäischen Vergleich. Das Genus des Substantivs. Mannheim: Institut für Deutsche Sprache. (= amades - Arbeitspapiere und Materialien zur deutschen Sprache 3/04).

Kalverkämper, Hartwig (1979): "Die Frauen und die Sprache". Linguistische Berichte 62: $55-71$.

Kelly, Louis G. (1969): 25 Centuries of Language Teaching. Rowley: Newbury House Publishers.

Klotz, Peter (2004): "Sprachreflexionskompetenz und kompetenter Sprachgebrauch". In: Kämper-Van den Boogart, Michael (ed.): Deutschunterricht nach der PISA-Studie. Frankfurt a. M. etc., Lang: 153-168.

Köpcke, Klaus-Michael (1982): Untersuchungen zum Genussystem der deutschen Gegenwartssprache. Tübingen: Niemeyer.

Köpcke, Klaus-Michael/Zubin, David A. (1996): "Prinzipien für die Genuszuweisung im Deutschen". In: Lang, Ewald/Zifonun, Gisela (eds.): Deutsch - typologisch. Berlin/New York, de Gruyter: 473-491. (= Jahrbuch des Instituts für Deutsche Sprache 1995).

Köpcke, Klaus-Michael/Zubin, David A. (1983): "Die kognitive Organisation der Genuszuweisung zu den einsilbigen Nomen der deutschen Gegenwartssprache". Zeitschrift für Germanistische Linguistik 11/1983: 166-182.

Köpcke, Klaus-Michael/Zubin, David A. (1984): "Sechs Prinzipien für die Genuszuweisung im Deutschen: Ein Beitrag zur natürlichen Klassifikation". Linguistische Berichte 93/84: 26-50. Reprint in: Sieburg, Heinz (ed.) (1997) Sprache - Genus/Sexus. Frankfurt a. M. etc., Lang: 86-115.

Krohn, Dieter/Krohn, Karin (2008): Der, das, die - oder wie? Studien zum Genuserwerb schwedischer Deutschlerner. Frankfurt a. M. etc.: Lang.

Leiss, Elisabeth (2005): "Derivation als Grammatikalisierungsbrücke für den Aufbau von Genusdifferenzierung im Deutschen". In: Leuschner, Torsten/Mortelmans, Tanja/De 
Groodt, Sarah (eds.): Grammatikalisierung im Deutschen. Berlin/New York, de Gruyter: 11-30. (= Reihe Linguistik - Impulse \& Tendenzen 9).

Lima, Elvira et al. (i. Vorb.): Wie viele Flexionsklassen verträgt die italienische Nominalflexion? Vorüberlegungen zu einer kontrastiven Beschreibung der Nominalmorphologie aus italienisch-deutscher Perspektive. (Erscheint demnächst im Rahmen des internationalen Projekts Eurogramm des Instituts für Deutsche Sprache Mannheim).

Meinert, Roland (1989): Die deutsche Deklination und ihre didaktischen Probleme. München: Iudicium.

Menzel, Barbara (2004): Genuszuweisung im DaF-Erwerb. Psycholinguistische Prozesse und didaktische Implikationen. Berlin: Weißensee.

Pusch, Luise F. (1979): "Der Mensch ist ein Gewohnheitstier, doch weiter kommt man ohne ihr. Eine Antwort auf Kalverkempers Kritik an Trömel-Plötz' Artikel über ,Linguistik und Frauensprache'". In: Linguistische Berichte 63: 84-102.

Putzer, Oskar (2004): "Grammatik im Spracherwerb". In: Di Meola, Claudio/Hornung, Antonie/Rega, Lorenza (2005): Perspektiven Eins, Akten der 1. Tagung Deutsche Sprachwissenschaften in Italien (Rom, 6.-7. Februar 2004). Roma, Istituto Italiano di Studi Germanici: 395-414.

fSieburg, Heinz (1997): "Einführung". In: Sieburg, Heinz (ed.): Sprache - Genus/Sexus. Frankfurt a. M. etc., Lang: 9-32.

Sperber, Horst G. (1989): Mnemotechniken im Fremdsprachenerwerb mit Schwerpunkt "Deutsch als Fremdsprache". München: Iudicium. (= Studien Deutsch 9).

Steinmetz, Donald (1986): "Two principles and some rules for gender on German: Inanimate nouns". Word 37: 189-217.

Thieroff, Rolf/Vogel, Petra M. (2009): Flexion. Heidelberg: Universitätsverlag.

Trömel-Plötz, Senta (1979): "Linguistik und Frauensprache". Linguistische Berichte 57: 49-68.

Ulrich, Miorita (1988): "'Neutrale' Männer - 'markierte' Frauen. Feminismus und Sprachwissenschaft." Sprachwissenschaft 13: 383-399.

Vester, Frederic (1975): Denken Lernen Vergessen. Stuttgart: dva.

Wegener, Heide (1995): "Das Genus im DaZ-Erwerb - Beobachtungen an Kindern aus Polen, Rußland und der Türkei". In: Handwerker, Brigitte (ed.): Fremde Sprache Deutsch. Tübingen, Narr: 1-24.

Wegera, Klaus-Peter (1996). Das Genus. Ein Beitrag zur Didaktik des DaF-Unterrichts. München: Iudicium.

Weinrich, Harald (2005). Textgrammatik der deutschen Sprache. 3. Auflage. Hildesheim: Olms.

Welte, Werner (1974): Moderne Linguistik: Terminologie/Bibliographie. 2 Bde., München: Hueber.

Werner, Martina (2008): Von analytisch zu synthetisch. Wie Genusmorpheme durch Syntax entstehen. Handout zum Vortrag anlässlich der 3. Tagung Deutsche Sprachwissenschaft in Italien, Rom, 14.-16. Februar 2008.

Zifonun, Gisela (2002): "Neue Wege der vergleichenden Grammatikbeschreibung". www.idsmannheim.de/gra/texte/zif2.pdf, Stand Juni 2010. Auch erschienen in: Ágel, Vilmos/Herzog, Andreas (eds.): Jahrbuch der ungarischen Germanistik 2001. Budapest/Bonn, Gesellschaft ungarischer Germanisten/DAAD: 143-155).

Zifonun, Gisela et al. (1997): Grammatik der deutschen Sprache. Band 1. Berlin/New York: de Gruyter. (= Schriften des Instituts für Deutsche Sprache 7.1).

Zubin, David A./Köpcke, Klaus-Michael (1981): "Gender - a less than arbitrary grammatical category". Chicago Linguistic Society 17: 439-449. 